\title{
Tra nubi, selve, giardini e tassonomie: nuova liuteria elettromeccanica ed elettroacustica nelle pratiche musicali di Mauro Lanza, Andrea Valle e Simone Pappalardo ${ }^{1}$
}

\author{
Luca Guidarini
}

Ricevuto il 13 Marzo 2021

Revisione del 4 Maggio 2021

\section{Introduzione: l'apparato strumentale}

Le ricerche presentate in questo articolo indagano le pratiche artistiche di Mauro Lanza, Andrea Valle e Simone Pappalardo, con particolare attenzione all'impiego concreto e alla definizione teorica degli strumentari di nuova liuteria elettromeccanica ed elettroacustica. Verranno presi in analisi i lavori Le Nubi non scoppiano per il peso (2011) di Mauro Lanza, il ciclo Selva Petrosa (2019-2020) di Andrea Valle, il ciclo Systema Naturae (2013-2017) di Andrea Valle e Mauro Lanza e la pratica performativa di Simone Pappalardo. Il lavoro di analisi e di studio delle componenti informatiche e degli apparati strumentali è stato possibile grazie al continuo confronto con i compositori, che mi hanno dato la possibilità di consultare e discutere insieme i materiali preparatori informatici alla base dei loro lavori. La mediazione informatica nella pratica compositiva è diventata, infatti, routine quotidiana e imprescindibile tanto nella strutturazione dell'opera musicale: dalla parametrizzazione automatizzata di qualità musicali, alla definizione dell'articolazione formale, alla determinazione dei singoli eventi, quanto nella creazione del complesso apparato strumentale nei casi indagati.

Con l'evoluzione della liuteria [...] lo strumento musicale mostra esplicitamente la sua natura di apparato: esso non coincide più con il solo oggetto d'artigianato che conosciamo in quanto tale (il violino, il flauto e i loro pari), ma comprende un impianto più complesso, costituito da ogni dispositivo necessario alla trasmissione e ricezione dei suoni prodotti ${ }^{2}$.

Come il compositore Giovanni Verrando evidenzia nell'articolo da cui è tratta la citazione riportata, un'evoluzione dell'indagine sulla nuova liuteria implica un ampliamento della visione dello strumento musicale, che da entità singola di produzione del suono e strettamente legata al gesto performativo umano, diventa apparato: una

${ }^{1}$ Supplementary materials related to this article are available online. The links are indicated accordingly.

2 G. Verrando, Gli strumenti come apparati, 2016. <https://www.giovanniverrando.net/lutheriecomposition/gli-strumenti-come-apparati/> 
molteplicità di parti che lavorano per produrre un determinato risultato timbrico nel caso qui indagato, astraendo la componente gestuale umana.

Gli apparati strumentali investigati da Lanza, Valle e Pappalardo sono costituiti da tre parti principali: un corpo sonoro elettromeccanico o elettromagnetico, un convertitore dal dominio digitale ad analogico, una macchina per la computazione.

Il computer, come macchina di controllo e computazione di dati, è in comunicazione con i dispositivi di controllo che fungono da convertitori dei dati digitali in azioni elettromeccaniche. I dispositivi di controllo presi in analisi nel presente studio sono di tre tipologie diverse: convertitori MIDI (in particolare https://www.interfacez.com), microcontroller (in particolare i controller Arduino, in grado di astrarre la componente computazionale dall'interfaccia del computer), e le schede audio.

Questo sistema complesso di produzione del suono rende possibile la sperimentazione personale - e casalinga - di pratiche computazionali che fanno riferimento a principi di robotica, physical computing e programmazione algoritmica. Come conseguenza di questo approccio tecnicizzato, si ha l'automazione di alcuni dei processi che portano allo sviluppo ultimo dell'opera musicale e si andranno ad approfondire nei prossimi paragrafi.

\section{Automazione e rumenta}

Tra gli elementi più urgenti che lo studioso inglese Aaron Bastani mette in evidenza nel suo manifesto Fully Automated Luxury Communism ${ }^{3}$ - che dà anche il nome ad un brano di musica elettronica di Mauro Lanza ${ }^{4}$ - c'è l'uso della tecnologia intesa come mezzo per emancipare la forza lavoro umana dalle operazioni meccaniche e semantiche ripetitive e continue, garantendo però una sostenibilità ecologica dei processi e dei mezzi di produzione: "The technologies need to deliver a post-scarcity, post-work society - centered around renewable energy, automation and information [...]»5.

Gli strumenti della nuova liuteria che si andranno a studiare nei prossimi paragrafi hanno in comune con questa visione socio politica della tecnologia la necessità di astrarre il lavoro meccanico del/la musicista, per automatizzare - con processi di computazione informatica - la produzione del suono, attraverso corpi risonanti di riciclo che, dicendola con $\check{Z}_{\text {ižek }}{ }^{6}$, si emancipano dalla loro ideologia e funzione primaria di mercato. Uno spremiagrumi, ideato, costruito e commercializzato con la funzione primaria di poter ottenere della rigenerante spremuta, ora viene controllato da un computer e il suo movimento si defunzionalizza del suo principale scopo di produzione, riappropriando la sua qualità sonora all'interno di una popolazione di strumenti simili a lui.

3 A. Bastani, Fully Automated Luxury Communism, Verso, Londra 2019.

${ }^{4}$ M. Lanza, Fully Automated Luxury Communism <https://matiere-memoire.bandcamp.com/album/ mmxx-14-fully-automated-luxury-communism>

5 A. Bastani, Fully Automated Luxury Communism, Verso, Londra 2019, p. 192.

6 S. Žižek (a cura di), Mapping Ideology, Verso, Londra 1994. 
Queste popolazioni di oggetti dal valore acustico riqualificato vengono indagate da Andrea Valle nella ricerca artistica sulle Residual Orchestras, dove l'aggettivo "residuale" acquisisce più valori semantici. Come Valle riporta", citando la definizione di "residual' fornita dall'Oxford Dictionary, i significati attribuiti al termine sono relativi al concetto di rimanenza, rimandando tanto a processi di degradazione materica, quanto alla natura dei dati derivati dalle procedure algoritmiche - specialmente gli errori da processi sperimentali. Si definisce quindi Orchestra Residuale il prodotto di una sperimentazione tecnica empirica che ha come obiettivo finale quello di creare tecnologie computazionali avanzate utilizzando strumenti riciclati, o low-cost. Questi processi computazionali, per comunicare con il mondo fisico che ci circonda, e quindi con il controllo degli apparati strumentali in analisi, si rifanno ai principi pratici tecnici di quello che viene definito physical computing. Questa pratica, nata agli inizi del 2000, è definita da Igoe e O'Sullivan nell'omonimo libro come la possibilità di operare processi computazionali al di fuori dell'interfaccia di un computer tradizionalmente inteso - comunque utilizzabile per la comunicazione e il controllo di dati - permettendo un arricchimento e una connessione dell'esperienza sensibile in relazione al dominio digitale. I primi esperimenti di Andrea Valle in questa direzione iniziano attorno al 2008, con il progetto Rumentarium: un'orchestra di 24 piccole percussioni automatizzate, utilizzata in modo estensivo in contesti improvvisativi e installativi, il cui nome deriva da rumenta, termine del Nord Italia per indicare la spazzatura ${ }^{8}$. La pratica musicale di riciclo di oggetti, tesa alla rivalutazione della componente di produzione sonora, tuttavia, per Valle risale agli anni '90 con sperimentazioni di musica concreta impiegando «materiali comuni, recuperati o autocostruiti»", affermandosi poi nel tempo come pratica di ricerca personale, per arrivare alle recenti composizioni per ensemble e strumenti automatizzati, come il ciclo Selva Petrosa.

Le Orchestre Residuali indagate da Valle sono accomunate da criteri di costruzione e scelta degli strumenti ben precisi e in linea con quanto detto: basso costo, competenze ridotte, semplicità di assemblaggio, robustezza e facile manutenzione, risoluzione temporale del suono strumentale ${ }^{10}$.

Anche Simone Pappalardo, accettando l'accezione terminologica "residuale", descrive tra le ricerche principali della sua pratica artistica il reimpiego di oggetti comuni, o la riqualifica acustica di oggetti riciclati. La nuova liuteria di Pappalardo, come si vedrà nel dettaglio, oltre agli strumenti elettromeccanici, integrerà degli strumenti la cui automazione acustica deriva da processi elettromagnetici.

L'automazione, nei casi qui studiati, non riguarda solamente la produzione di un elemento sonoro, ma coincide anche con l'elaborazione dell'impianto articolativo-for-

${ }^{7}$ A. Valle, Residual orchestras: Notes on low profile, automated sound instruments, in Proceedings of the Cumulus Conference '15, McGraw-Hill, Milano 2015, pp. 717-729.

8 A. Valle, Making Acoustic Computer Music: The Rumentarium project, in Organised Sound, 18(03), Cambridge 2013, pp 242-254.

9 <http://www.thenewnoise.it/i-regnum-di-andrea-valle-e-mauro-lanza>

10 A. Valle, Residual orchestras: Notes on low profile, automated sound instruments, in Proceedings of the Cumulus Conference '15, McGraw-Hill, Milano 2015, pp. 717-729. 
male di ogni singolo lavoro. Mauro Lanza infatti, come più volte dichiarato ${ }^{11}$ utilizza l'ambiente di programmazione OpenMusic ${ }^{12}$ per elaborare su più livelli l'intero processo compositivo, coincidente con la scrittura di un preciso algoritmo volto a mettere in azione un determinato processo musicale. Andrea Valle utilizza SuperCollider ${ }^{13} \mathrm{con}$ un approccio simile. Per Valle la programmazione dell'apparato algoritmico coincide con l'intero progetto compositivo, arrivando in certi casi a determinare automaticamente lo stadio simbolico della partitura finale. Per Mauro Lanza questo avviene solo parzialmente: il prodotto degli algoritmi tesi alla generazione di un contenuto simbolico di OpenMusic vengono a loro volta manipolati al fine di orchestrarli manualmente, $\mathrm{o}$ - nei casi in cui l'algoritmo elabora la totalità delle informazioni del livello simbolico necessarie per la partitura - direttamente dal trascrittore Luca Valli con cui Lanza lavora a stretto contatto $^{14}$.

Nel caso di Pappalardo, operando in contesti prettamente improvvisativi e installativi, le soluzioni algoritmiche automatizzate sono sviluppate in Max/MSP${ }^{15}$ in funzione di un controllo parametrico dello strumento - dove l'azione performativa è determinata dal compositore stesso - nel primo caso, e nel secondo caso con sistemi di network adattivi basati sul principio del feedback, tra gli strumenti stessi. Un sistema adattivo, nella definizione che ne dà Claudio Panariello ${ }^{16}$, «è un sistema capace di modificare le sue variabili interne in funzione dei suoi input, al fine di soddisfare un compito"; nel caso degli apparati strumentali di Simone Pappalardo quindi, i principi adattivi determinano aspetti formali e di articolazione dell'opera.

\section{Questioni organologiche}

L'insieme di pratiche (computazione informatica, controllo algoritmico, nuova liuteria da oggetti di riciclo) che vanno a costruire l'apparato strumentale della nuova liuteria elettromeccanica di Lanza, Valle e Pappalardo, tendono a sfuggire ad un approccio di classificazione incentrato solamente sul mezzo meccanico di produzione del suono e le sue qualità acustiche, come quelli dell'organologia critica della scuola di Hornbostel e Sachs. La quinta classe della classificazione degli strumenti introdotta nel 1940 da

11 Sono reperibili online diverse masterclass del compositore dove illustra il suo impiego di OpenMusic, tra le più recenti: <https://www.facebook.com/793985660723793/videos/396656014901634>; o dal seminario dell'IRCAM del $2013 \mathrm{https://medias.ircam.fr/xc203e0 \_ pre769sentation-de-la-pie768ce-ludus.}$

$12<$ https://openmusic-project.github.io/>

$13<$ https://supercollider.github.io/>

${ }^{14}$ Come riportato da Ingrid Pustijanac in Oralità 'digitalizzata' nelle edizioni del repertorio contemporaneo, in "Convegno: Filologia musicale e tecnologie digitali a colloquio con la prassi esecutiva", Fondazione Guido d'Arezzo, Arezzo/online 2020, nonché da una breve comunicazione personale da parte del compositore.

$15<$ https://cycling74.com/products/max>

16 C. Panariello, Study in three phases. An Adaptive Sound Installation, in «Leonardo Music Journal», 30, 2020, pp. 44-49. 
Curt Sachs ${ }^{17}$, ovvero quella degli strumenti elettrofoni, sembra risolvere parzialmente la questione classificatoria. In Galpin lo strumento elettrofono viene così definito:

Electronic instruments or electric vibrators, i.e. instruments in which the sound-waves are formed by oscillations set up in electric valves. This class, sometimes called electronic, is entirely new and included here for the first time. It is only recently that the familiar "howl" of the oscillating valve has been raised to the dignity of musical expression. Electromagnetism is also used ${ }^{18}$.

Chiaramente nel 1937 Galpin si poneva il problema della classificazione di un nuovo strumento la cui energia vibrante non è più dipendente dall'azione umana, bensì dalla presenza o meno della corrente elettrica utile per azionare le valvole indispensabili per la produzione del suono nei primordiali strumenti elettrici. Secondo Kartomi questo non implica un cambiamento strutturale nella suddivisione proposta da Hornbostel e Sachs, bensì un'ulteriore sottocategorizzazione di alcuni elementi. Riportando l'esempio della chitarra elettrica, Kartomi evidenzia come le sue qualità organologiche rimangono invariate rientrando nella classe dei cordofoni, ma esplicitando la necessità dell'amplificazione per il completo funzionamento dello strumento. Andrea Valle e Mauro Lanza ${ }^{19}$ mettono in luce i criteri di classificazione dell'apparato strumentale analizzato nel paragrafo 4.3, indispensabili per l'intero processo compositivo del ciclo Systema Naturae, che, come si vedrà, si basa strutturalmente su diverse dimensioni di classificazione. La sistematizzazione degli strumenti proposta dai compositori impiega le cinque classi di Hornbostel e Sachs (idiofoni, membranofoni, cordofoni, aerofoni ed elettrofoni) applicate al corpo sonoro, suddividendone la modalità di controllo, ma senza classificare l'intero apparato strumentale. La dettagliatissima suddivisione dello strumentario proposta da Valle e Lanza può essere interpretata quindi come una sub-classificazione ricorsiva della quinta classe di Sachs: tutti gli apparati strumentali qui presentati infatti sono vitalmente dipendenti da un circuito elettrico, necessario per l'attivazione del preciso gesto meccanico e automatizzato, che agisce su un corpo sonoro ben preciso, classificabile a sua volta secondo le classi di Hornbostel e Sachs. Accettando infatti le riflessioni di Giulia Accornero sulla classificazione della nuova liuteria ${ }^{20}$, un'organologia critica sulla nuova liuteria non può rimanere imbrigliata in un sistema tassonomico chiuso e non ampliabile. Considerando quindi le prospettive dinamiche dell'applicazione di Hornbostel e Sachs, si accetta il termine "elettromeccanico" per strumenti la cui messa in vibrazione acustica è de-

17 Teorizzata inizialmente dallo studioso inglese Francis Galpin, e introdotta in C. Sachs, The History of Musical Instruments, Norton, New York 1940

18 F. Galpin, A Textbook of European Musical Instruments. William, London 1937.

19 A. Valle, M. Lanza, Systema naturae: shared practices between physical computing and algorithmic composition, in J. P. Tapio Lokki and V. Välimäki (a cura di), «Proceedings of the 14th Sound and Music Computing Conference», Aalto University, Espoo 2017, pp. 391-398.

${ }^{20}$ G. Accornero, Un'organologia critica per una nuova liuteria, in G. Manca, L. Manfrin (a cura di), Fare Strumento. Composizione, invenzione del suono e nuova liuteria, Edizioni ETS, Pisa 2018. 
terminata da un movimento meccanico con l'energia di attivazione dipendente dalla presenza della corrente elettrica. Nel caso di Simone Pappalardo si parlerà anche di strumenti elettromagnetici: le tecniche da lui implementate prevedono infatti l'attivazione di corpi vibranti attraverso la creazione e la manipolazione algoritmica di campi elettromagnetici.

\section{Dentro le pratiche musicali di Mauro Lanza, Andrea Valle e Simone Pappalardo}

Per entrare nel dettaglio degli apparati strumentali e delle pratiche compositive di Lanza, Valle e Pappalardo verranno affrontati alcuni esempi tratti dalla produzione musicale dei tre compositori, con il supporto materiale di esempi, immagini, video e software forniti dai compositori stessi, permettendo così il confronto diretto con le fonti per l'indagine sulle tecniche compositive impiegate nelle opere in analisi, e consistono in Le nubi non scoppiano per il peso di Mauro Lanza, Selva Petrosa di Andrea Valle, Systema Naturae di Lanza e Valle, e Millis() di Simone Pappalardo.

La molteplicità dei materiali e delle fonti in esame ${ }^{21}$ consente di indagare la questione della nuova liuteria elettromeccanica ed elettromagnetica sotto il punto di vista costruttivo, di controllo computazionale e di formalizzazione nell'opera musicale. Per ogni progetto in analisi si presenteranno quindi le qualità organologiche degli strumenti automatizzati, il loro controllo e le tecniche di gestione algoritmica del materiale musicale.

Nei casi di Selva Petrosa, Systema Naturae e per gli strumenti sviluppati da Simone Pappalardo, i compositori stessi interverranno attraverso dei video dimostrativi delle tecniche di volta in volta analizzate.

\subsection{Le nubi non scoppiano per il peso: la macchina della pioggia di Mauro Lanza}

Le nubi non scoppiano per il peso, del 2011 per ensemble, soprano di coloratura, elettronica e macchina per la pioggia, rappresenta il primo brano di Mauro Lanza compositore veneziano formatosi all'IRCAM di Parigi - che include un apparato strumentale elettromeccanico affiancato alla compagine umana, e in questo caso anche quella elettronica sintetica. Non è il primo brano però in cui il compositore veneziano sperimenta con oggetti quotidiani, di recupero o capaci di produrre suoni altamente stereotipati: già in Barocco del 1998 e Vesperbild del 2006-2007 introduce degli strumenti giocattolo - con il loro caratteristico suono impuro, la cui interfaccia strumentale è tesa ad uno scopo ludico infantile - all'interno degli ensemble. Così come

21 I materiali e le tematiche affrontate sono frutto di una serie di interviste e comunicazioni personali via mail o chat avvenuti tra i mesi di novembre 2020 e febbraio 2021 con i compositori stessi. Oltre al confronto diretto, di grande importanza per questo studio hanno avuto le numerose pubblicazioni scientifiche e divulgative di Andrea Valle, gli articoli e gli studi che sono stati svolti su Mauro Lanza, le interviste online svolte negli anni precedenti reperibili per Lanza, Valle e Pappalardo, e i materiali inediti che sono stati messi a disposizione di chi scrive. 
nel successivo Ludus de Morte Regis, del 2013 per coro ed elettronica, ai/lle cantanti sono affidati una grande quantità di piccoli strumenti dal timbro percettivamente identificabile e culturalmente affermato, come: fischietti, campanelli, giocattoli sonori e palloncini petofoni. Le flatulenze, infatti, sono prese da Lanza come esempio di un suono con una sua propria identità semantica, viste come oggetti sonori con le proprie qualità timbriche da essere inseriti in un contesto sonoro più ampio, utilizzando anche il mezzo elettronico per facilitarne l'integrazione ${ }^{22}$.

Le nubi non scoppiano per il peso è una commissione di Ensemble Court-Circuit e "Integra - Fusing music and technology", progetto europeo del Conservatorio di Birmingham, ed eseguito in prima assoluta l'11 settembre 2011 al Festival Ultima di Oslo, Norvegia. Come espresso dal compositore nelle note di programma, il brano "deals with heaviness and fall, and with measuring what seems to have no measure» ${ }^{23}$. Il testo, intonato dalla soprano, e derivato dal biblico Libro di Giobbe, si interroga sulla nascita dei fenomeni naturali, chiedendosi «Ha forse un padre la pioggia? E chi mette al mondo le gocce di rugiada?». Con il dispositivo da lui ideato - e realizzato con il tecnico Simon Cacheux e con la supervisione preliminare di Thierry Coduys - Mauro Lanza diventa idealmente il "padre" generatore di queste gocce, comandandone la caduta. Ispirata dall'installazione sonora Dripper01 di Arno Fabre ${ }^{24}$ la struttura ideata da Lanza e Cacheux consiste in un contenitore di legno dalle dimensioni di $35 \times 35 \times 60 \mathrm{~cm}$, al suo interno una tanica da riempire con l'acqua, il dispositivo di controllo isolato, e sedici interruttori elettromagnetici che operano il rilascio dell'acqua attraverso altrettanti tubi di silicone, affissati ad una struttura di metallo (Fig.1).

Il dispositivo di controllo consiste in un convertitore dal dominio digitale a quello analogico che opera via MIDI, collegandosi alle due porte della scheda audio (in partitura è indicato come riferimento il modello RME Fireface 800). Una scheda audio dalle caratteristiche simili a quella consigliata è necessaria non solo per le porte MIDI, ma anche per il corretto funzionamento dei file audio della componente elettronica, con frequenza di campionamento e quantizzazione a $96 \mathrm{kHz}$ e $24 \mathrm{bit}$.

La componente computazionale dei dati MIDI è gestita da più software con diverse funzioni: una patch di Max/MSP (aggiornata al runtime 5) che controlla singolarmente i componenti della struttura viene impiegata per il test, l'installazione, il fine-tuning e lo svuotamento della struttura; per la performance i dati midi sono controllati dalla DAW Logic Pro 9, che gestisce allo stesso tempo il click generato con il plugin Kontakt, i cinque file audio della componente elettronica, e il riverbero a convoluzione di cinque oggetti amplificati.

La struttura in metallo viene sospesa a circa 2.6 metri dal tavolo posto sotto di essa, e i tubi distano circa 2 metri dalla tanica d'acqua. Sotto ognuno dei tubi in silicone, sopra il tavolo sottostante alla struttura, vengono posti degli oggetti sonori attivati dalle gocce stesse che cadono (Fig. 2). Questi oggetti consistono in:

\footnotetext{
$22<$ https://vimeo.com/244839789>

$23<$ https://brahms.ircam.fr/works/work/31028/>

$24<$ http://arnofabre.free.fr/en/Dropper01/Dropper01.html>
} 


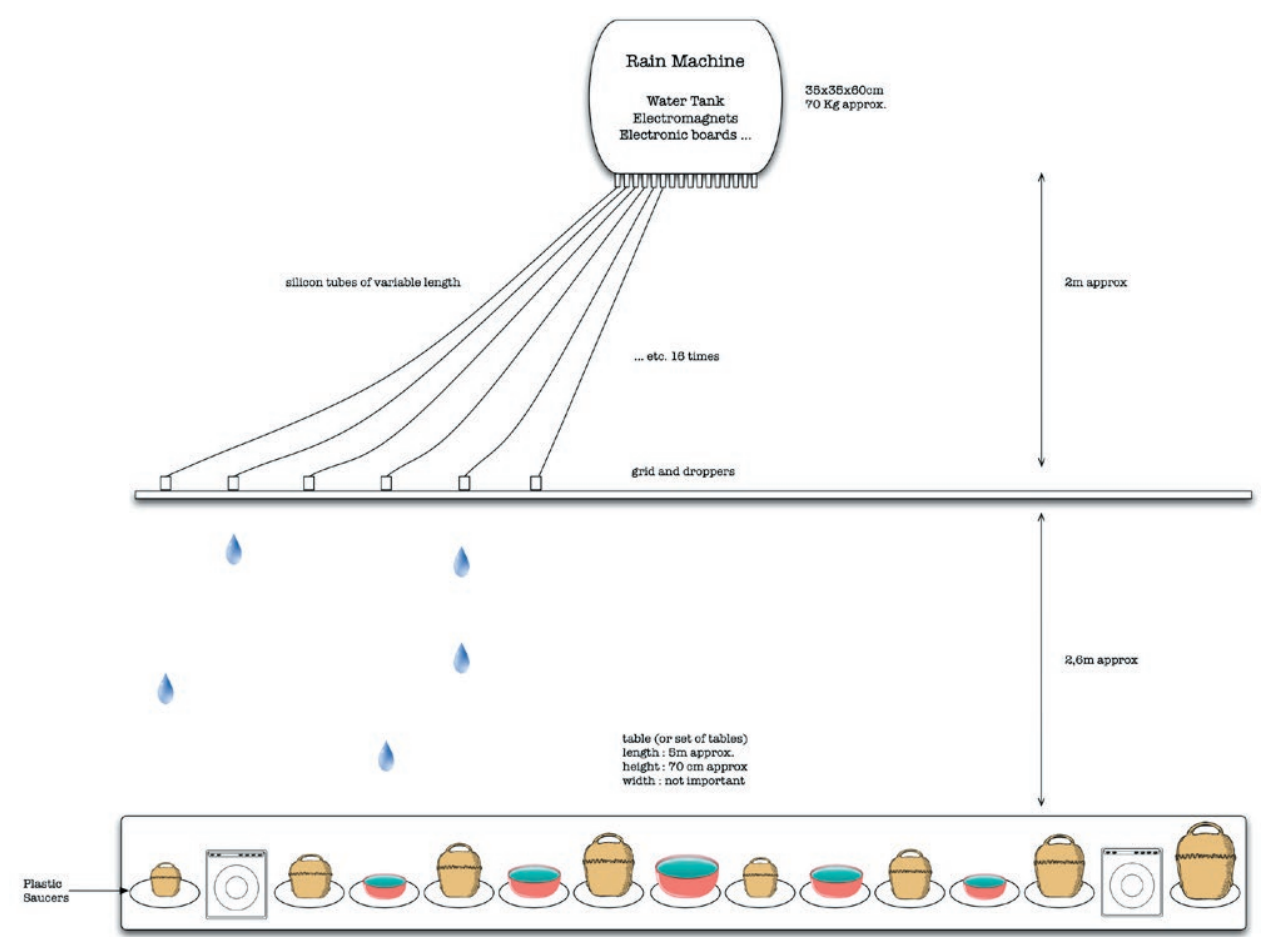

Figura 1. M. Lanza, Le nubi non scoppiano per il peso, Ricordi, Milano 2011.

- cinque recipienti o scodelle. Lanza suggerisce di usare campane tibetane, scodelle di latta o ciotole cinesi. Il suono in questo caso consiste nel riempimento progressivo della ciotola con le gocce; i contenitori devono essere leggermente riempiti d'acqua prima della performance;

- otto oggetti molto risonanti intonati (nell'ordine da sinistra a destra, Mib4, Si3, Sol\#3, Sol3, Do\#4, La3 - 440 hz - Fa\#3 e Mib3). Il compositore suggerisce di impiegare dei campanacci intonati, indicando come l'intonazione e la risonanza debbano essere le qualità da considerare nel momento della scelta degli oggetti;

- due piastre elettriche, tipo fornello da campeggio. Da attivare poco prima della performance, non al massimo della potenza e inclinandole leggermente. La goccia cadendo sulla piastra emette il classico suono dell'evaporazione su una superficie incandescente.

Ogni oggetto, ad eccezione delle piastre elettriche, deve essere posizionato a sua volta sopra una piccola struttura autocostruita utilizzando dei vasi larghi da giardino e degli elastici. Appoggiati a questo supporto, gli strumenti vibreranno più liberamente, una volta messi in vibrazione dalle gocce d'acqua.

In partitura è indicato come questi quindici oggetti debbano essere minuziosamente microfonati, amplificati ed equalizzati, utilizzando un mixer dedicato. I microfoni impiegati sono dei piezoelettrici ceramici sui recipienti e sugli oggetti risonanti, mentre 


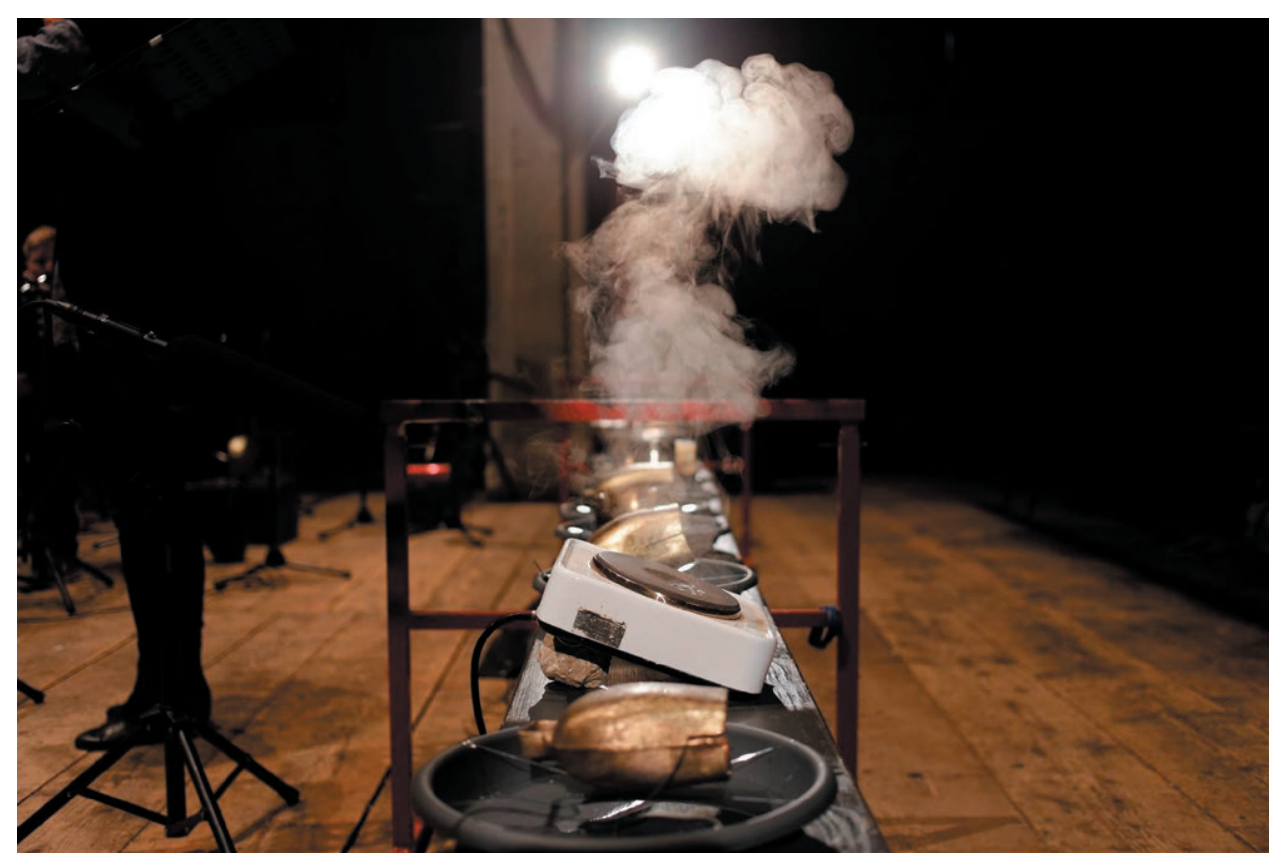

Figura 2. Microfonazione e posizionamento degli strumenti risonanti di Le nubi non scoppiano per il peso, (CPablo Fernandez, Festival Les Amplitudes, 2018.

per le piastre elettriche sono consigliati due microfoni direzionali di tipo shotgun. I singoli oggetti devono a loro volta essere equalizzati, per essere affinati timbricamente ed evitare che interferiscano frequenze esterne e disturbanti rispetto a quelle interessate.

Formalmente il brano si configura in due macro-sezioni che esplorano due idee musicali differenti. La prima - dall'inizio a battuta 309 - esplora la creazione, l'articolazione e lo sfasamento di diverse figure macro ritmiche. La seconda macrosezione - da battuta 310 alla fine del brano - è frutto delle ricerche condotte da Lanza sulla sintesi per modelli fisici, e del loro riversamento nella scrittura musicale per ensemble. Entrambe le sezioni sono composte utilizzando il linguaggio di programmazione OpenMusic, utilizzato con due prospettive differenti.

Per la prima sezione Lanza ha immaginato il cadere delle gocce come dei suoni impulsivi di un metronomo che si materializza dal nulla (Fig.3), che gradualmente va a comporre dei moduli ritmici grazie all'orchestrazione del suo graduale sfasamento (Fig.4). Questo graduale sfasamento porta alla costruzione di moduli macro ritmici che vengono ripetuti tre volte (Fig. 5), fino ad arrivare all'omoritmia delle battute 292-299, evaporando nelle scale ascendenti di battute 300-309 che concludono la macro sezione.

La seconda parte del brano sviluppa algoritmicamente gli elementi sonori a partire dalla sintesi per modelli fisici di tubi messi in eccitazione dal rumore bianco. I musicisti dell'ensemble impiegano, soffiandoci dentro, dei tubi di plastica zigrinata, dal diametro di $16 \mathrm{~mm}, 41.5 \mathrm{~cm}$ e $49.4 \mathrm{~cm}$ al clarinetto; $21.3 \mathrm{~cm}, 35 \mathrm{~cm}$ e $44 \mathrm{~cm}$ al 


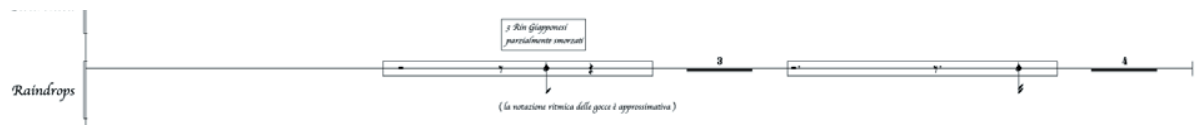

Figura 3. M. Lanza, Le nubi non scoppiano per il peso, Ricordi, Milano 2011, p. 1.

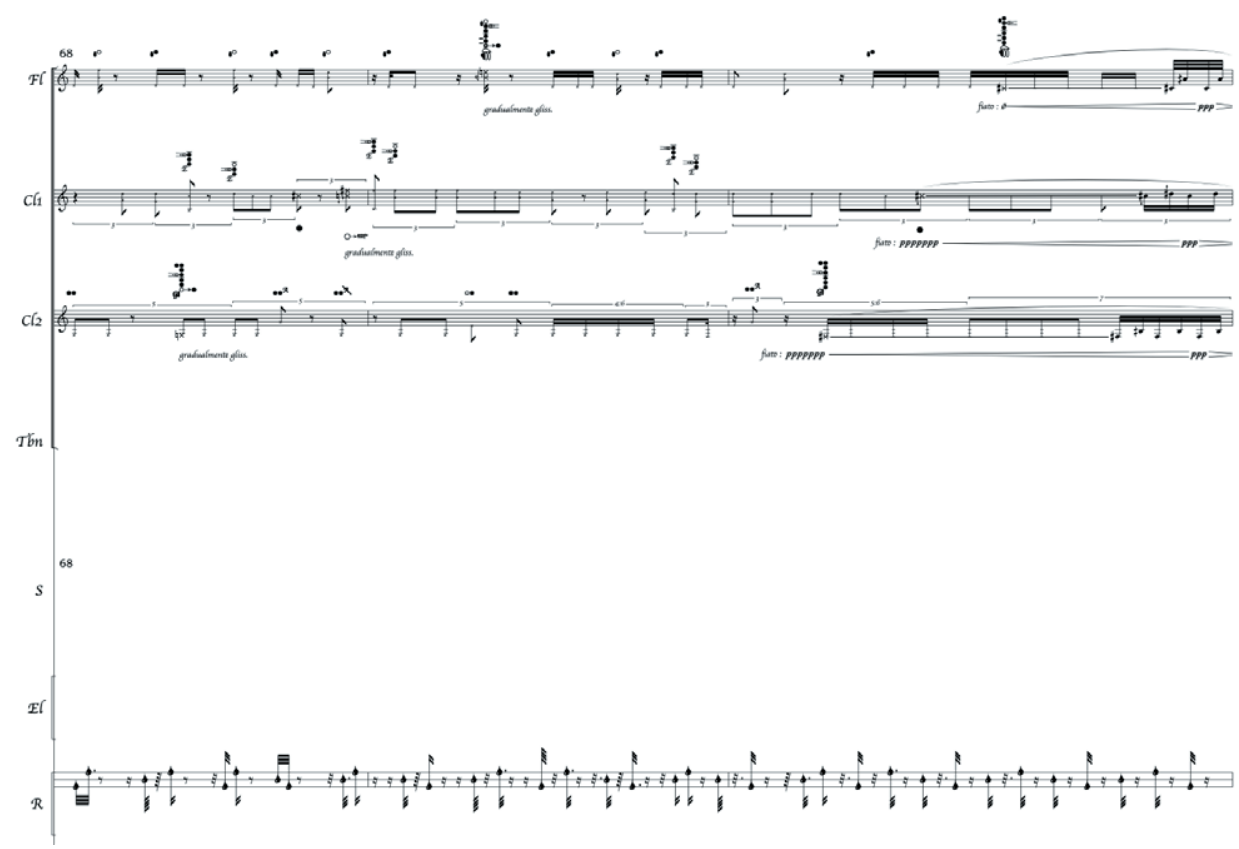

Figura 4. M. Lanza, Le nubi non scoppiano per il peso, Ricordi, Milano 2011, p. 11.

trombone basso; $39.2 \mathrm{~cm} 45 \mathrm{~cm}$ e $52.3 \mathrm{~cm}$ alle percussioni; $46.6 \mathrm{~cm}$ al pianoforte. La sintesi per modelli fisici ${ }^{25}$ prende per assunto la possibilità di calcolare un segnale sulla base di equazioni che descrivono il comportamento acustico e meccanico dei corpi che agiscono nella sua produzione, definendo algoritmicamente parametri quali il volume dell'oggetto modellato, la densità e il tipo di materiale, il modo e

25 «La synthèse par modèles physiques est une technique de synthèse sonore qui, au lieu de s'attacher à reproduire le son lui-même (par l'analyse des fréquences qui le compose par exemple), part du dispositif physique producteur de ce son. Ainsi, cette technique se démarque des méthodes de synthèse digitale comme la synthèse soustractive, additive, etc. L'objectif de cette synthèse est autant scientifique qu'artistique car celle-ci permet aux scientifiques d'approfondir leur connaissance des phénomènes sonores et offre aux musiciens un nouveau matériau pour la composition» [La sintesi per modelli fisici è una tecnica di sintesi del suono che, invece di cercare di riprodurre il suono stesso (analizzando le frequenze che lo compongono, per esempio), parte dal dispositivo fisico che produce il suono interessato. Così, questa tecnica differisce dai metodi di sintesi digitale come la sintesi sottrattiva e additiva, ecc. L'obiettivo di questa sintesi è tanto scientifico quanto artistico perché permette agli scienziati di approfondire la loro conoscenza dei fenomeni sonori e offre ai musicisti un nuovo materiale per la composizione.], A. Hufschmitt, La synthese par modeles physiques, tesi di dottorato, Université de Paris Sorbonne (Paris IV) U.F.R. de Musique et Musicologie, Parigi 2000, pp. 11-13. 

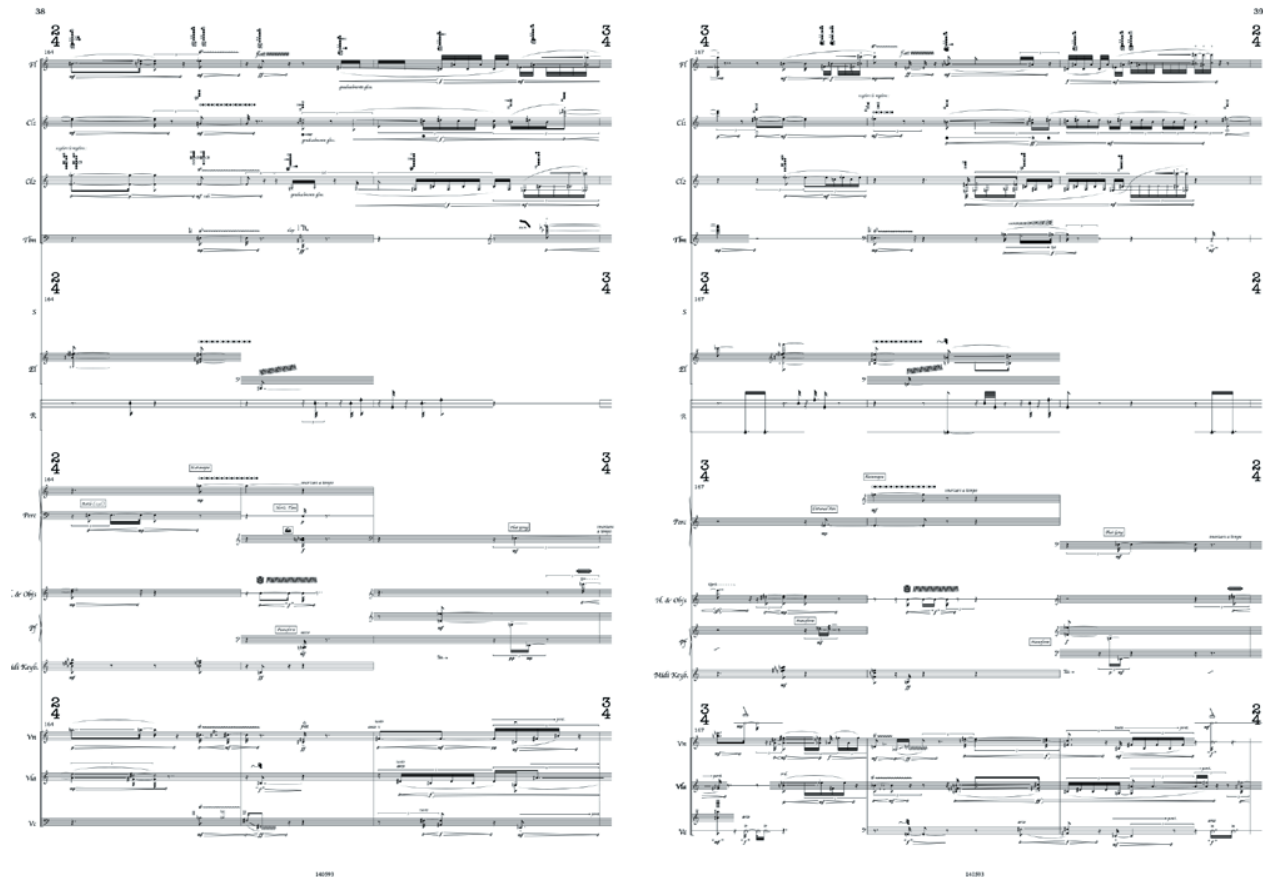

Figura 5. M. Lanza, Le nubi non scoppiano per il peso, Ricordi, Milano 2011, pp. 38-39.

la pressione di eccitazione, la sua lunghezza e la sua forma. Mauro Lanza ha sviluppato questo tipo di sintesi con il software Modalys, da lui approfondito durante il cursus dell'IRCAM ${ }^{26}$.

Modalys, inizialmente con il nome Mosaic, viene sviluppato all'IRCAM fin dal $1991^{27}$ e si rivela di grande interesse per le compositrici e i compositori del circuito ircamiano. Come Foglia riporta, Mosaic viene utilizzato da Saariaho fin dal 1992, per la generazione dei suoni di sintesi di Amers e Près, per violoncello ed elettronica ${ }^{28}$. Nel caso di Saariaho, Mosaic era in comunicazione con PatchWork - l'antecedente di OpenMusic ${ }^{29}$ - con una struttura costruita appositamente. Dopo un periodo di disuso all'interno dell'IRCAM, come Lanza riporta, durante la sua permanenza del 1999 all'istituto parigino, il compositore veneziano ha avuto modo di studiarlo approfonditamente, e diventando negli anni successivi il docente del software all'interno

26 M. Lanza, note di sala del concert-atelier monografico dedicato a Mauro Lanza, IRCAM, Espace de projection, Parigi, 13/02/2004.

27 A. Hufschmitt, La synthese par modeles physiques, tesi di dottorato, Université de Paris Sorbonne (Paris IV) U.F.R. de Musique et Musicologie, Parigi 2000.

28 C. Foglia, Ristrutturazione spettrale di armonie funzionali: l'evoluzione della forma teleologica in Kaija Saariaho, tesi di laurea magistrale, Università di Pavia - Dipartimento di Musicologia e Beni Culturali, Cremona 2020, p. 189, e nel convegno Spectralisms all'IRCAM del $2019<\mathrm{https://medias.ircam.fr/x4179ef>}$

29 G. Assayag, C. Rueda, Computer-Assisted Composition at IRCAM: From PatchWork to OpenMusic, «Computer Music Journal», vol. 23, no. 3, 1999, 59-72. 
del cursus. La compositrice Paola Livorsi, parlando del suo brano Os del 2001 per voce maschile ed elettronica ${ }^{30}$, mette in luce i processi compositivi delle applicazioni di Modalys in OpenMusic, sottolineando come questo fosse possibile grazie alla libreria OM Modalys 1.0 (poi sviluppata in una seconda versione), implementata e approfondita da Mauro Lanza. A partire dal 2019 l'IRCAM ha messo a fruizione gratuita per gli iscritti al forum online il kernel di Modalys aggiornato al runtime 3.6.0, le librerie implementate per OpenMusic, Max/MSP e Matlab, oggi impiegate anche da Lanza ${ }^{31}$. La libreria personale di Mauro Lanza, contrariamente a quelle distribuite dall' istituto parigino, permetteva l'esecuzione di operazioni multithreading, mentre quella distribuita oggi esegue thread singoli. L'ambiente di Lanza permette inoltre un'ulteriore comunicazione con l'ambiente di programmazione CSound via terminale, per elaborare la sintesi in tempo differito, creando i file score e orchestra automaticamente dai dati di sintesi in linguaggio LISP di OpenMusic.

Come esprime Lanza nelle già menzionate note di programma di Erba nera tu cresci segno nero tu vivi, durante la programmazione degli algoritmi di sintesi per modelli fisici, nonostante la complicata componente computazionale, l'interesse e l'idea centrale rimangono quelle dello strumento musicale, in questo caso creato digitalmente: «Le compositeur se fait à la fois luthier et interprète de sa proprie musique»" ${ }^{32}$ Questa visione del compositore come "liutaio digitale", con Le nubi non scoppiano per il peso si ritrova anche al dominio elettromeccanico, con la macchina della pioggia, applicando criteri già sperimentati nel dominio digitale.

\subsection{Selva Petrosa: dentro la Selva di Andrea Valle}

Il modus operandi di un liutaio, impegnato nella costruzione di un apparato strumentale complesso, controllabile attraverso un computer, dal suono instabile, inarmonico e "impuro" e derivato da materiali di recupero, come si è già avuto modo di discutere poco sopra, è tra gli interessi di ricerca di Andrea Valle. Bassista elettrico di estrazione rock e improvvisativa, compositore allievo di Azio Corghi, ricercatore e docente presso l'Università di Torino e il Conservatorio della stessa città, vanta di un corpus molto vasto di produzione artistica e di pubblicazioni scientifiche. Addottorato in semiologia all'Università di Bologna, ha all'attivo, tra le altre, pubblicazioni sulla notazione musicale ${ }^{33}$,

30 C. Agon, J. Bresson, e G. Assayag (a cura di), The OM Composer's book vol. 1, Editions Delatour France/IRCAM, Parigi 2006, pp. 133-146

$31<$ https://forum.ircam.fr/projects/detail/modalys/>; la documentazione aggiornata è consultabile $<$ http://support.ircam.fr/docs/Modalys/current/co/publication-web.html>; la ricerca è del gruppo S3AM team (Sound Systems and Signals: Audio/Acoustics, InstruMents) <https://www.ircam.fr/recherche/ equipes-recherche/systemes-et-signaux-sonores-audioacoustique-instruments-s3am/>

${ }^{32}$ M. Lanza, note di sala del concert-atelier monografico dedicato a Mauro Lanza, IRCAM, Espace de projection, Parigi, 13/02/2004.

33 A. Valle, La notazione musicale contemporanea. Aspetti semiotici ed estetici, De Sono-EDT, Torino 2002 . 
sull'informatica musicale ${ }^{34}$, e sulla programmazione con SuperCollider ${ }^{35}$. La poliedrica attività artistica, oltre alle produzioni qui esaminate, si confa di installazioni sonore (si ricordano qui l'installazione performativa del 2014 Otosimbionte, con Simone Pappalardo e le installazioni interattive Rumentario autoedule, Organo fonatorio e Macchina logotelica del 2012), brani per ensemble di improvvisatori (come il disco Cortège d'Alsaxy), sonorizzazioni di film muti (si ricorda la recente commissione del Museo del Cinema di Torino per la sonorizzazione de Il Jockey della Morte di Alfed Lind, e Maciste di Giovanni Pastrone), set di improvvisazione con basso solo e dispositivi elettromeccanici, e dischi autoprodotti di diversa estrazione ${ }^{36}$. Questo insieme di pratiche si coniuga nell'interesse del compositore per la generazione algoritmica di parametri musicali. Come afferma in un'intervista del 2018, alla base dei suoi interessi sulla composizione algoritmica si ritrovano due motivazioni principali:

D'abord, j'admire cette possibilité qu'ont les poètes et les peintres de commencer une œuvre immédiatement, de faire un premier geste et de pouvoir le poursuivre... Mais en musique, c'est différent, il n'y a pas isomorphisme. Il y a forcément une médiation entre le geste et le résultat. J'ai souvent essayé de travailler avec des logiciels pour avoir une relation plus directe, mais à la fin cela ne fonctionnait pas vraiment. Cela ne marche pas car la résolution temporelle de la notation est très différente de la résolution temporelle du geste. Ou bien on part de la partition, ou bien on part du geste. La deuxième raison est liée à mon parcours issu de l'improvisation, que je trouve très liée à l'algorithmique par son utilisation des patterns, de la mémoire de la forme, des procédures devant fonctionner en temps réel, des variations... autant de méta-décisions qui sont très algorithmiques. C'est peut-être paradoxal, mais improvisation et algorithmique me semblent intimement liés dans cette idée de procédure ${ }^{37}$.

L'interesse per una risposta gestuale-musicale immediata, guidata da processi algoritmici, ha portato il compositore piemontese a sviluppare una fruttuosa e continua ricerca attorno alle orchestre residuali, come definite nel secondo paragrafo. A partire dal primo Rumentarium, Valle infatti arriva a definire diverse orchestre residuali di varia natura, che vengono di volta in volta adattate alle necessità e ai contesti artistici.

34 A. Valle, V. Lombardo, Audio e Multimedia, Maggioli, Santarcangelo di Romagna 2014 (ed. orig. Apogeo, Milano 2002).

35 A. Valle, Introduzione a SuperCollider Apogeo Education - Maggioli Editore, Milano 2015.

36 https://andreavalle.bandcamp.com/

37 Prima di tutto, ammiro questa possibilità dei poeti e dei pittori di iniziare un lavoro immediatamente, di fare un primo gesto e di poterlo continuare... Ma nella musica è diverso, non c'è isomorfismo. C’è necessariamente una mediazione tra il gesto e il risultato. Ho provato spesso a lavorare con i software per avere un rapporto più diretto, ma alla fine non ha funzionato. Non funziona perché la risoluzione temporale della notazione è molto diversa dalla risoluzione temporale del gesto. O si parte dallo spartito, o si parte dal gesto. La seconda ragione è legata al mio background nell'improvvisazione, che trovo molto legata alla pratica algoritmica per il suo uso di pattern, memoria della forma, procedure che devono lavorare in tempo reale, variazioni... tante meta-decisioni che sono molto algoritmiche. Può essere paradossale, ma l'improvvisazione e l'algoritmo mi sembrano intimamente legati in questa idea di procedura.] <http:// musiquealgorithmique.fr/entretien-6-andrea-valle/> 
Questo tipo di approccio - in sviluppo e in continua espansione organologica - viene definito dallo stesso compositore come "Punk Munari". Nelle parole di Valle:

Ho posto questo approccio sotto l'emblema "Punk Munari". Da un lato, Massimo Banzi, uno dei fondatori del progetto Arduino ha sollecitato un approccio punk alla tecnologia: ovvero il provare anche se non si sa, in termini inglesi "learning by doing", secondo un modo punk di intendere l'approccio alla musica ("questi sono degli accordi: ora fai una canzone"). Dall'altro, in Codice ovvio Bruno Munari ha sottolineato l'importanza dello sperimentare e del definire una teoria dell'oggetto in parallelo alla sua stessa costruzione: un approccio che il designer milanese chiama "TMO", ovvero "Tecnica mentre opero" 38 .

Queste inclinazioni hanno portato quindi Valle a sperimentare personalmente le possibilità di comunicazione tra il mondo fisico e quello digitale offerte dalle tecniche di physical computing, fino a declinare delle soluzioni personali per il controllo del dominio acustico alternative rispetto a quelle illustrate da Igoe e O'Sullivan ${ }^{39}$. Il compositore e ricercatore torinese più volte rivendica quindi una pratica di "acoustic computer music", controllando gli apparati strumentali elettromeccanici con tecniche derivate dalle esperienze storiche di computer music algoritmica: «je ne cherche pas à construire un instrument électronique pouvant être manipulé, sinon pourquoi ne pas utiliser directement un ordinateur?» ${ }^{40}$.

In particolare, nel paragrafo presente e in quello successivo, verranno trattate due tecniche di generazione algoritmica di dati parametrici musicali a partire dalla rappresentazione digitale di un fenomeno sonoro: la "cartonificazione spettrale" 41 di un segnale audio come modello compositivo per l'esempio di Selva Petrosa, e la tecnica dell'“audioparismo", approfondita nell'esempio di Isimiosia papanabuis del paragrafo seguente.

Per modello sonoro "cartonificato" si intende un'analisi spettrale semplificata ma coerente con i principi generali. Questo tipo di analisi è stata implementata da Andrea Valle con l'architettura SonaGraph, sviluppata in SuperCollider, illustrata dal compositore stesso e riprodotta in fig. 6 .

L'idea di analisi spettrale basata su un banco di filtri è sviluppata fin dagli anni Venti del Novecento all'interno dei laboratori Bell, con la problematica della non corrispondenza della fase del segnale. L'algoritmo digitale del Phase Vocoder, contrariamente, interpreta una Trasformata di Fourier operando con un banco di filtri che con-

$38<$ https://www.musicaelettronica.it/come-costruire-un-serraturofono-a-borracce-programmabilee-interattivo-guida-teorico-pratica-1/>

39 Andrea Valle, cercando soluzioni pratiche a problemi derivati dall'utilizzo intensivo dei controller Arduino Uno, teorizza quella che viene definita "Audio physical computing", e consiste nel convertire il segnale audio in segnale elettrico, utile per il controllo degli elementi fisici. Per maggiori dettagli: A. Valle, Audio physical computing, in SMC Sound \& Music Computing Conference proceedings 2011, Padova 2011.

$40<\mathrm{http}$ //musiquealgorithmique.fr/entretien-6-andrea-valle/>

41 Dall'inglese "cartoonified spectral model". Il termine è introdotto da D. Rocchesso, F. Fontana (a cura di), The Sounding Object, Edizioni di Mondo Estremo, 2003. 


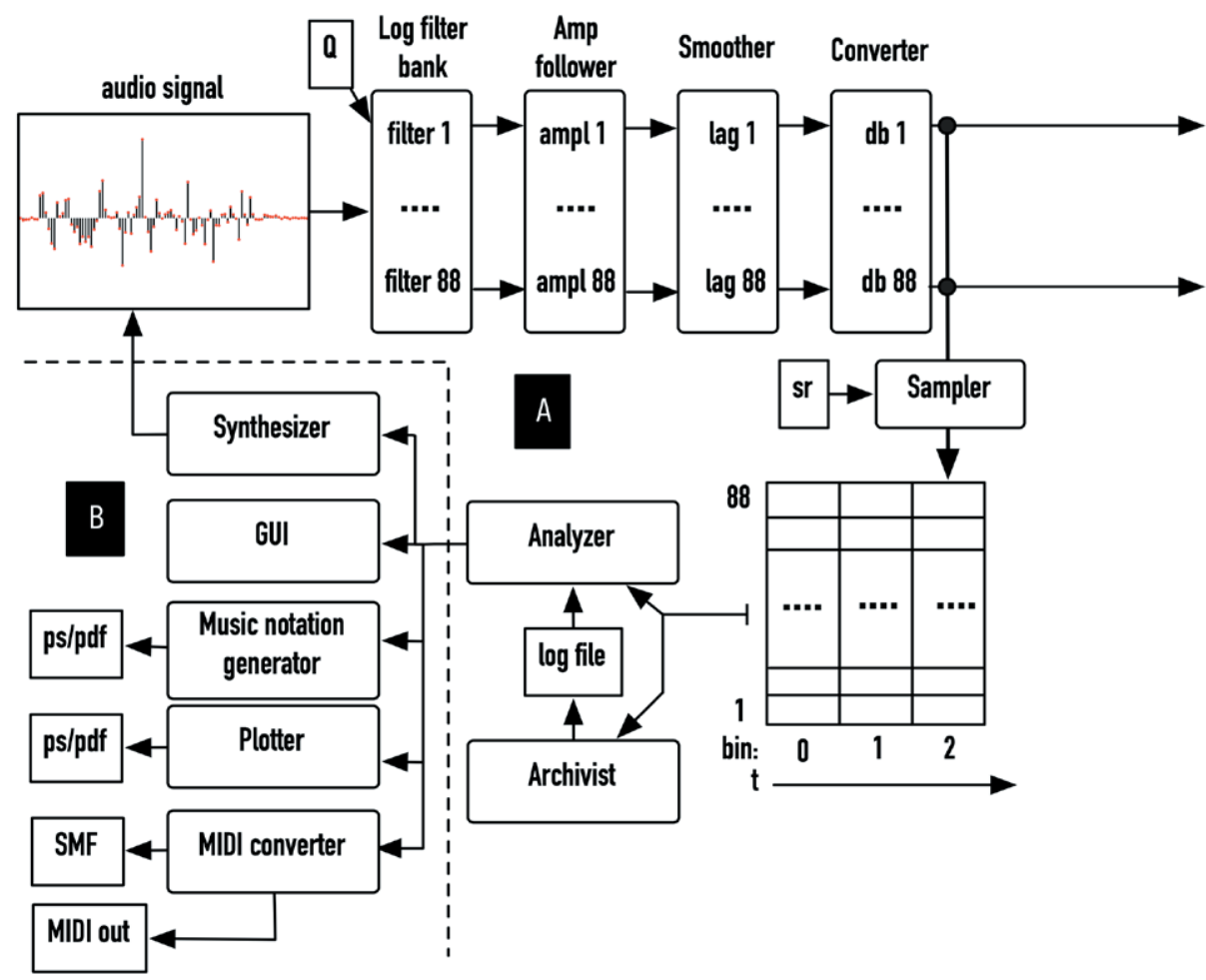

Figura 6. (CA. Valle, architettura di SonaGraph, Sonagraph. A cartoonified spectral model for music composition, in SMC Sound \& Music Computing Conference proceedings 2011, Málaga 2019.

sidera la fase del segnale. Nonostante questo, come fa notare Valle ${ }^{42}$, la fase del segnale è indispensabile per la risintesi di un segnale audio, ma può essere messa in secondo piano nel momento in cui si effettua un'operazione di estrazione di dati analitici.

Il livello analitico del segnale (in figura indicato come Log filter bank) è attuato da un banco di 88 filtri passa-banda Constant- $Q$, in cui la frequenza centrale è accordata in modo logaritmico in relazione ad un'astrazione della tastiera di un pianoforte. L'accordatura dei filtri e la loro ampiezza è variabile; Valle ha optato al compromesso degli 88 filtri ordinati secondo logiche strumentali per ragioni di risoluzione delle frequenze e dimensione del banco di filtri, andando a coprire una fascia spettrale che va dai $27.5 \mathrm{~Hz}$ ai $4186 \mathrm{~Hz}$. Seguendo il modello del phase vocoder (precisamente l'algoritmo di encoding sviluppato per il Vocoder di Dudley ${ }^{43}$ ), ogni filtro è connesso a un envelope follower (in Fig. 6 Amp follower), la cui uscita è a sua volta smussata utilizzando un filtro passa-basso, per eliminare variazioni troppo rapide di dati (in fig. 6 Smoother). Da questa operazione si ottiene una chiara formalizzazione dei dati di

42 A. Valle, Sonagraph. A cartoonified spectral model for music composition, in SMC Sound \& Music Computing Conference proceedings 2011, Málaga 2019.

43 Ibid. 
variazione di ampiezza, a scapito delle informazioni sulla fase. Ad ogni segnale audio quindi, una volta filtrato e smussato, i valori di ampiezza del suono vengono convertiti da lineari a logaritmici (in Fig. 6 Converter), venendo poi campionati ad una frequenza di campionamento stabilita (in Fig. 6 Sampler). La risoluzione temporale della finestra spettrale è determinata dalla frequenza di campionamento e può essere regolata a seconda dei fini dell'analisi. Ogni singolo campione viene a sua volta memorizzato all'interno di una matrice bidimensionale. Ogni riga di questa matrice rappresenta una singola istanza temporale, e contiene i singoli valori del filtro alla data frequenza di campionamento.

Questa tecnica fin dal momento del suo sviluppo, è stata impiegata da Valle in diverse situazioni compositive, ed in particolare ne ha fatto un uso intensivo nel recente Selva Petrosa, ciclo di quattro composizioni per strumenti tradizionalmente intesi e strumenti elettromeccanici. Le singole composizioni del ciclo (ad oggi ne sono state composte tre, la quarta è in fase germinale), nominate Selva Petrosa (2019, per violoncello e pianoforte e strumenti elettromeccanici, su commissione di mdi ensemble), Nova Selva Petrosa (2020, per chitarra elettrica, fisarmonica e tiorba e strumenti elettromeccanici, su commissione di azione_improvvisa ensemble), e Terza Selva Petrosa (2020, per chitarra elettrica e strumenti elettromeccanici, su commissione del chitarrista Salvatore Sorrentino), sono a loro volta composte da otto brani, derivati da analisi di brani rock degli anni ' $90^{44}$ con SonaGraph. Sono stati analizzati quindi lavori dei gruppi: Primus, Faith no More, Soundgarden, Rollins Band, Living Colour, Voivod, Melvins, Alice in Chains, Pantera, Negazione, Helmet, Jane's Addiction, Anthrax, Suicidal Tendencies, Fluxus e Pixies.

Per quanto riguarda l'apparato strumentale automatizzato, gli strumenti elettromeccanici utilizzati nell'intero ciclo consistono in un cetro tenore, un cetro basso, e un serraturofono a bottiglie organizzati come in fig. 7 .

La trasmissione dei dati avviene attraverso la comunicazione via rete tra il computer e un Arduino Mega, che controlla i 24 motorini dei cetri e le dodici serrature elettroniche del Serraturofono a borracce.

I due cetri, realizzati con l'aiuto della liuteria Alvermann, consistono in due cetre da tavolo con delle corde da basso, con un dispositivo di plettraggio a rotazione con un motorino da $12 \mathrm{~V}$. Il controllo è discreto, e comandato via Arduino.

Il Serraturofono a borracce è uno strumento a percussioni intonate, dove il corpo risonante consiste in dodici borracce di alluminio intonate cromaticamente, anche in questo caso il controllo è discreto e comandato via Arduino ${ }^{45}$. Le ragioni che hanno portato il compositore verso le borracce di alluminio come risonatori sono molteplici: per le qualità sonore quando percosse; per la loro risonanza; per la facilità di intonazione utilizzando acqua; per la facile reperibilità e il basso costo; per la semplicità di assemblaggio e la facile trasportabilità.

${ }^{44}$ Nell'intervento autobiografico Notes on a Minor Composer of the Italian Baroquesotto lo pseudonimo Andreas von Tal, il compositore chiama la musica rock con il termine ironico "petrosa".

45 Per un riferimento sulla programmazione di Arduino, <https://www.musicaelettronica.it/comecostruire-un-serraturofono-a-borracce-programmabile-e-interattivo-guida-teorico-pratica-3/> 


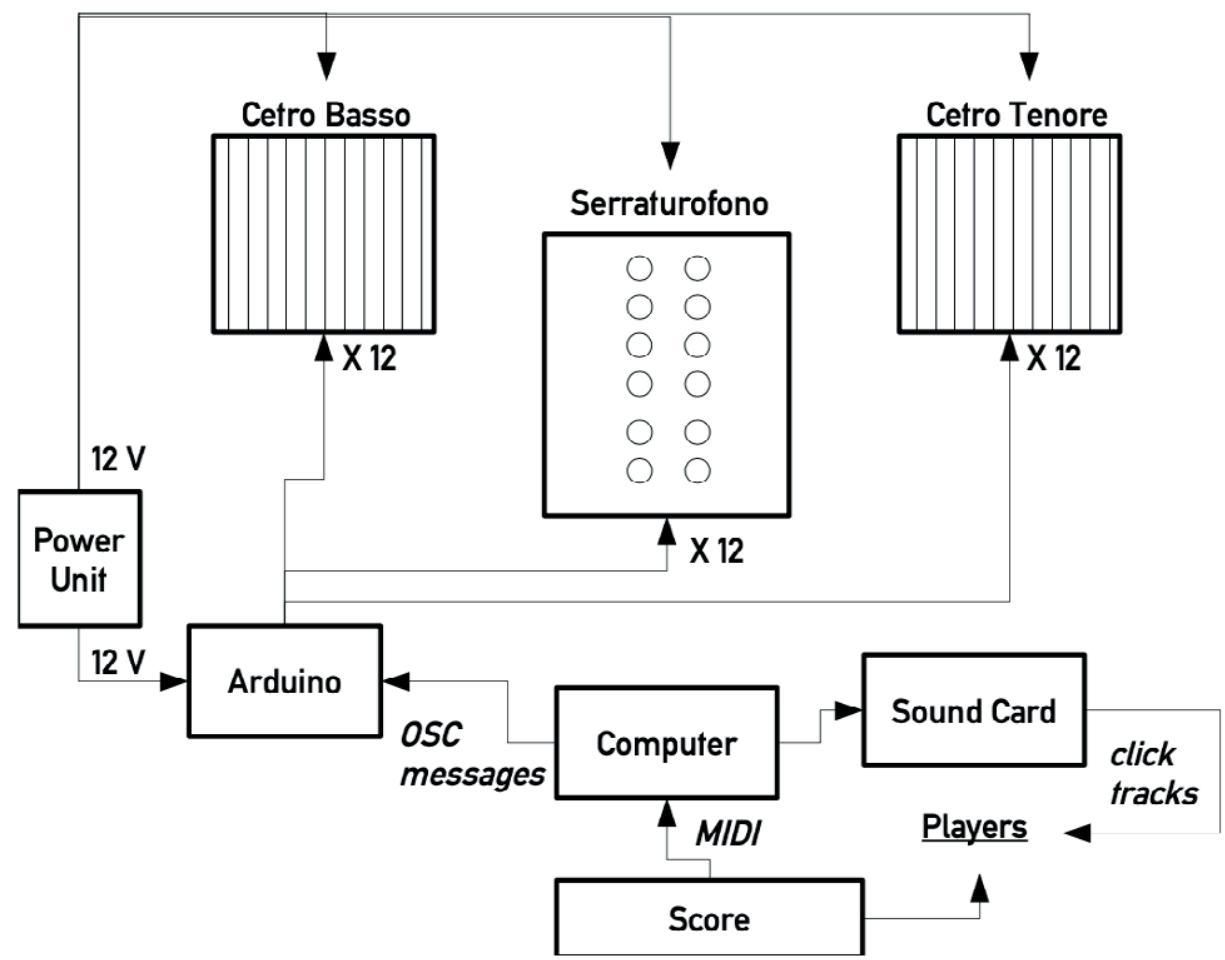

Figura 7. (CA. Valle, Selva Petrosa. Disposizione degli strumenti e delle interfacce elettroniche.

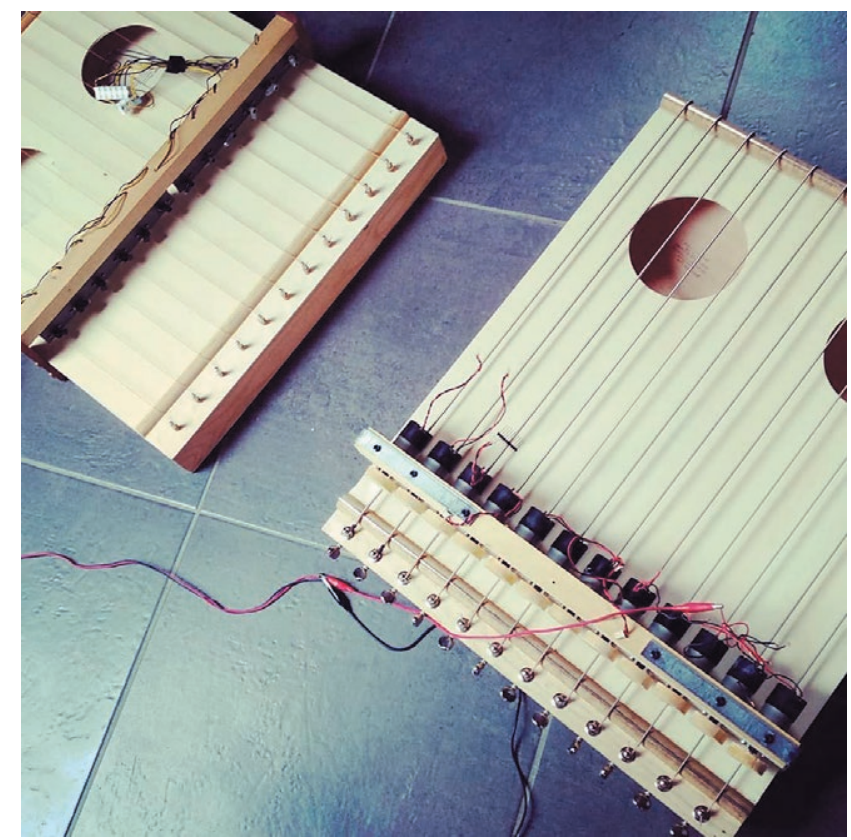

Figura 8. (CA. Valle, 2019. In foto i due cetri. 


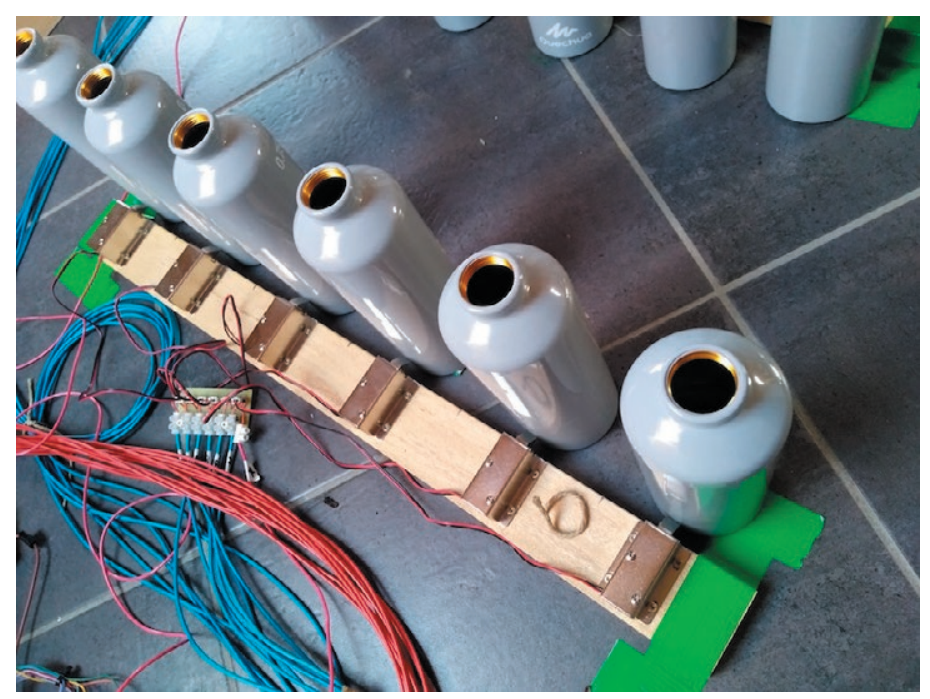

Figura 9. (CA. Valle, 2019. Serraturofono a borracce.

La risonanza delle borracce - scelte dopo un lungo processo di ricerca e sperimentazione - viene di volta in volta accordata empiricamente con dell'acqua, per ottenere una scala cromatica di 12 suoni; nel caso di Selva petrosa l'estensione va da Mib4 a Re5.

Le borracce sono messe in eccitazione da serrature elettriche controllate via Arduino. Mentre gli attuatori lineari via solenoide non presentano un meccanismo di ritorno del cilindro alla fine del movimento - avendo bisogno di un dato di rientro una serratura elettrica risolve il problema incastonando il solenoide in una gabbia (con la possibilità di un suo inserimento su una superficie), possedendo un range ben determinato e un voltaggio a $12 \mathrm{~V}$, ideale per operare nel mondo del physical computing. Le serrature meccaniche vengono quindi avvitate su una superficie di legno alla quale vengono fissate le borracce nella posizione di risonanza.

In Video 1, Andrea Valle approfondisce alcuni aspetti costruttivi dell'ensemble elettromeccanico, entrando nel dettaglio delle tecniche compositive impiegate per Selva petrosa, in particolare per Alai Nwedi, primo brano tratto dalla Terza Selva Petrosa per chitarra elettrica e strumenti elettromeccanici.

[Video 1 https://zenodo.org/record/4761448]

\subsection{Systema Naturae: animali automatizzati, algoritmi e pratica condivisa}

Systema Naturae è un ciclo di quattro composizioni dalla forma a catalogo, formate a loro volta da una serie di brevi brani per Orchestre Residuali ed ensemble di varia natura, nato come lavoro a quattro mani di Mauro Lanza e Andrea Valle su commissioni di RepertorioZero, Ensemble mosaik e Ensemble 2e2m. Il titolo prende ispirazione 
dall'omonima opera tassonomica di Linneo ${ }^{46}$, e come si vedrà nel dettaglio, il concetto di tassonomia e classificazione si presenta a più livelli del processo compositivo. Le quattro composizioni a loro volta fanno ancora riferimento alle tre categorie tassonomiche illustrate da Linneo, più una quarta immaginata dai compositori. Sempre da Linneo, i due compositori hanno preso inoltre la pratica della nomenclatura binomiale, che caratterizzerà i titoli dei brevi brani di Regnum Animale e Regnum Vegetabile. La prima composizione del ciclo è Regnum Animale, del 2013, per violino, viola, violoncello e strumenti elettromeccanici, è una commissione di RepertorioZero e Milano Musica. Come suggerito dal nome, il primo Regnum ciclo metaforizza la categoria degli esseri animali indagata da Linneo, e vede un'orchestra residuale di 25 oggetti quotidiani e obsoleti di recupero. Regnum Vegetabile è la seconda composizione del ciclo, del 2014 e commissionato dall'ensemble mosaik, e prende come metafora il secondo gruppo tassonomico di Linneo: il mondo vegetale. L'ensemble umano si compone di flauto, oboe, clarinetto, violino, viola e violoncello; mentre l'Orchestra Residuale in questo caso consiste in 30 asciugacapelli automatizzati. Il terzo brano è Regnum Lapideum e si riferisce al mondo inanimato delle pietre di Linneo, scritto nel 2016, su commissione dell'ensemble $2 \mathrm{e} 2 \mathrm{~m}$, per flauto, sassofono, pianoforte, percussioni, chitarra, viola e violoncello, e uno strumentario elettromeccanico di 25 strumenti che indaga i suoni impulsivi. Fossilia, ultima composizione del ciclo e commissione congiunta di ensemble mosaik, RepertorioZero e Ensemble $2 \mathrm{e} 2 \mathrm{~m}$, consiste in una quarta categoria immaginata dai due compositori, che metaforizza la riduzione allo stato di fossile, evocando i concetti di morte e quindi di fine, di tutte le categorie di Linneo precedentemente illustrate. Da suonare in coda ai tre Regna, consiste concretamente nell'unione degli organici strumentali (sia umani ed elettromeccanici), che vengono suonati insieme.

Nonostante la vicinanza storica dalla scrittura del ciclo, la documentazione scientifica e informativa pubblicata e reperibile su Systema Naturae consiste in un piccolo ma considerevole corpus che affronta l'opera sotto diverse prospettive, spesso mettendo in rapporto lo strumentario implementato da Lanza e Valle con un laboratorio barocco di produzione di strumenti inusuali.

Giacomo Albert ${ }^{47}$ mette in luce le relazioni che si affermano tra performance umana e macchina automatizzata, tracciando due orizzonti storiografici sulla sound sculpture e sulla composizione algoritmica. Partendo da un esempio storico di automazione meccanico nell'ambito musicale - il Giardino Armonico di Athanasius Kircher - arrivando con Systema Naturae a definire il concetto di "hypermedia", per sottolineare l'individualità artificiale di ogni singolo corpo risonante automatizzato e per contestualizzare la loro vivezza in relazione all'ambiente musicale e performativo.

Esemplificando brani da Regnum Animale, Albert constata come siano presenti tecniche compositive e modelli di articolazione che derivano dalla storia della musica

46 Carl von Linné, italianizzato in Carlo Linneo (1707-1778), è teorico, botanico e naturalista svedese, ha pubblicato Systema Naturae nel 1735.

47 G. Albert, 'Sound sculptures' e 'sound installations', «AAA - TAC», 7, Fondazione Giorgio Cini Venezia, Istituto per la musica, Fabrizio Serra editore, Roma-Pisa 2010. 
occidentale, come gli Hochetus in Ioris casachocii. L'ibridazione di queste esperienze compositive con i dispositivi elettromeccanici per l'autore comporta: "the counterbalancing of the process of de-materialization of content into procedures with its opposite, the re-materialization of procedures into many objects (either physical or cultural) which keep their own individuality» ${ }^{48}$, dove il processo di dematerializzazione del materiale nel tempo è collegato con quello di "media converge" e "media transcoding" illustrati in Lev Manovich ${ }^{49}$.

Daniela Fantechi, diversamente, riflette sull'atto compositivo teso alla sperimentazione sul materiale organologico che si ripercuote risultato ultimo della composizione, e sull'applicazione della "acoustic computer music" come mezzo di questa sperimentazione, soffermandosi infine sugli aspetti di organizzazione della partitura e del sistema di classificazione implementato dai due compositori ${ }^{50}$.

Systema Naturae, ed in particolare i brani Ioris Casachocii- approfondendo le analisi iniziate da Albert - e Zampychis flalutengla, rispettivamente i numeri XI e IV da Regnum Animale, vengono presi come esempio da Amy Bauer in diverse occasioni, all'interno di indagini analitiche sulla musica di Mauro Lanza ${ }^{51}$. I brani sopra citati, analizzati nella loro componente simbolica e performativa, vengono ricontestualizzati dall'autrice - insieme a Omysomyomys cacaca, Adius geradii, e Urophoturonta glistrispus - nella composizione di Anatra Digeritrice, per orchestra, del 2013.

Il ciclo Systema Nature viene inoltre presentato da Andrea Valle e Mauro Lanza in diverse occasioni (articoli divulgativi, pubblicazioni scientifiche, masterclass e lezioni di composizione), rappresentando per entrambi un dispensario di numerose tecniche compositive sviluppate con la pratica della scrittura musicale di natura algoritmica.

Nel già citato articolo a quattro mani ${ }^{52}$, i due compositori parlano della pratica compositiva condivisa, avvenuta principalmente attraverso la comunicazione e lo scambio di informazioni online, esemplificato in Fig. 10.

Secondo i compositori, il lavoro può essere suddiviso in tre fasi: design dell'architettura, organizzazione degli elementi sonori, progettazione della performance. La prima fase consiste nella definizione dei materiali preparatori: dai parametri organizzativi e formali, alla costruzione e preparazione degli strumenti. La seconda fase consiste nel processo che inizia con la strutturazione iniziale degli algoritmi fino alla formalizzazione della partitura. Valle e Lanza in questo momento della pratica si scambiano informazioni, algoritmi, diagrammi, grafici, registrazioni e simulazioni audio. In particolare,

48 Albert p. 124.

49 Lev Manovich, The Language of New Media, MIT Press, Cambridge Mass., 2001.

50 D. Fantechi, Systema Naturae, by Andrea Valle and Mauro Lanza, experimentation as starting point of a piece of "acoustic computer music", "Proceedings of the Electroacoustic Music Studies Network Conference», Firenze 2018.

51 A. Bauer, Automata in extremis: Mauro Lanza's sublime sound machines, "Nuove musiche», no. 5, 2021; <https://medias.ircam.fr/x58ad83>

52 A. Valle, M. Lanza, Systema naturae: shared practices between physical computing and algorithmic composition, in J. P. Tapio Lokki and V. Välimäki (a cura di), "Proceedings of the 14th Sound and Music Computing Conference», Aalto University, Espoo 2017, pp. 391-398. 


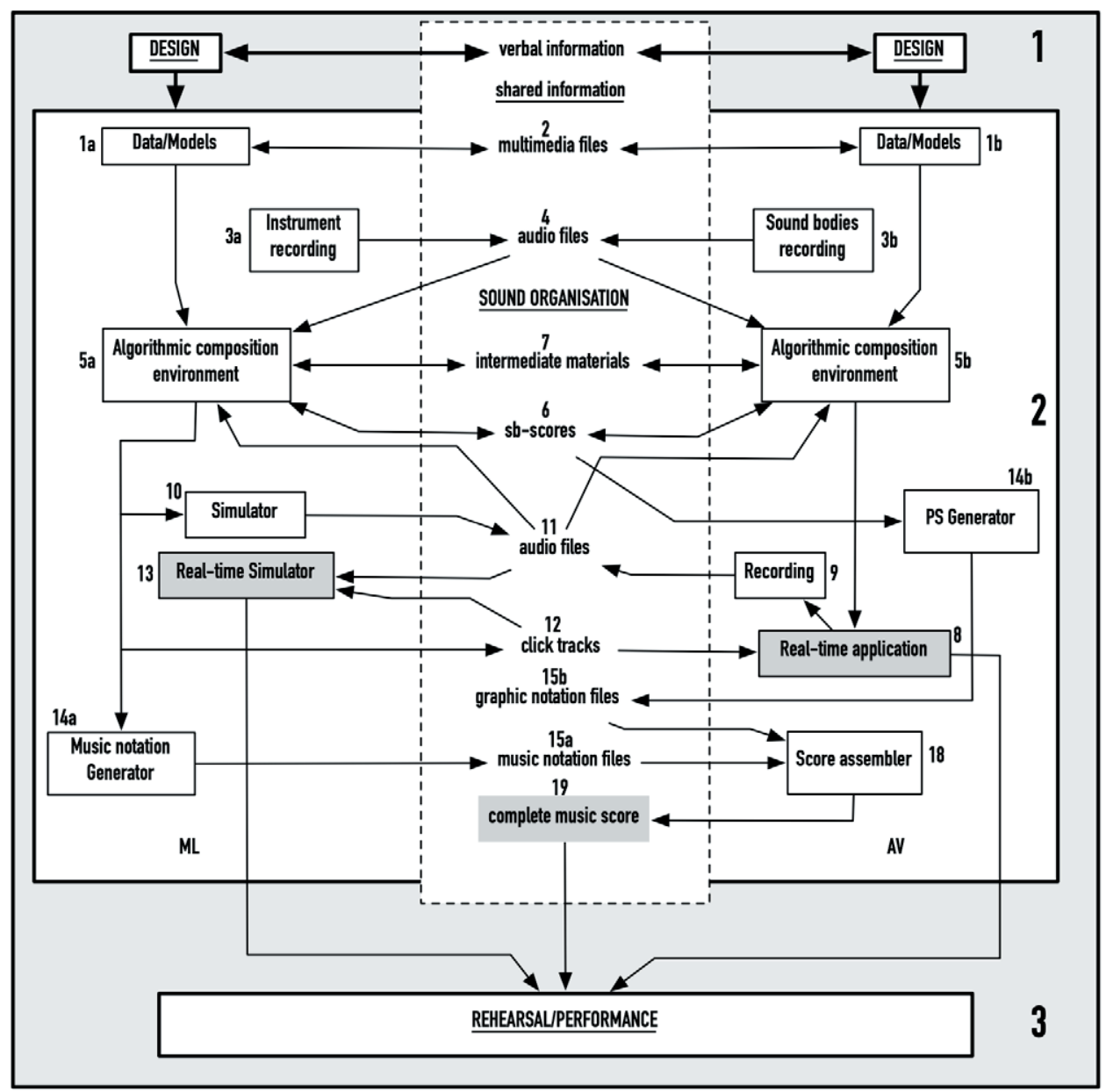

Figura 10. (CA. Valle, M. Lanza, Regnum Lapideum, inedito 2016. Disposizione degli strumenti.

la comunicazione di dati e informazioni compositive tra gli ambienti di programmazione utilizzati dai due compositori (OpenMusic per Mauro Lanza e SuperCollider per Andrea Valle) è permessa grazie alla creazione di un linguaggio informatico condiviso, chiamato Sb-score e simile ad un file score di Csound ${ }^{53}$. Il formato è quindi il seguente:

sirenetto1 60.62520 .0670255

zampogno5 21.889220 .05218182127

Ogni riga specifica un evento grazie al nome dello strumento, il tempo di inizio e durata in secondi dell'evento, valori iniziali e finali come interi con una risoluzione di 8 bit. Naturae.

53 Le liste complete nel formato implementato si trovano in appendice ad ogni Regna di Systema 
Il livello di formalizzazione della partitura si muove su due livelli che convergono allo stadio finale: su OpenMusic viene generato il livello notazionale degli strumenti tradizionali; su SuperCollider viene esportato un file in formato PostScript con la notazione grafica degli strumenti elettromeccanici. In ultima istanza l'intera partitura viene assemblata automaticamente da quello chiamato in Fig. 10 "Score assembler", per poi convertirne il risultato in formato PDF.

La terza fase del lavoro compositivo, così come illustrato da Valle e Lanza, coincide con l'elaborazione della componente performativa, sviluppando l'intera architettura di controllo - software e hardware - necessaria per l'esecuzione dal vivo.

La componente fisica strumentale automatizzata, per l'intero ciclo, consiste in un totale di 88 strumenti elettromeccanici, suddivisi a loro volta in "famiglie" nei tre regna (Fossilia vede l'utilizzo di tutto lo strumentario dei brani precedenti).

Dal punto di vista esecutivo, SuperCollider comunica direttamente con le porte digitali di Arduino, operando in tempo reale, seguendo le partiture digitali che permettono uno stretto sincronismo con la traccia click, e quindi con i musicisti.

All'atto pratico gli strumenti sono costruiti, con l'aiuto di Francesco Richiardi, in modo tale che i loro componenti possano essere sostituiti on the run in caso di danneggiamento in sede di prove con l'ensemble, come ha testimoniato Valle.

L'indagine sulle specifiche tecniche su cui si vuole riflettere nel presente articolo non può che partire dall'illustrazione dello strumentario di Systema naturae e le sue caratteristiche definite brano per brano. Solo in un secondo momento si potrà mettere queste informazioni in dialogo con la dimensione di gestione algoritmica del materiale musicale. Per ogni caso sarà infine fornito un video in cui sarà il compositore stesso a illustrare passaggi fondamentali del processo compositivo sull'esempio del caso specifico scelto.

In Regnum Animale le "famiglie" in cui vengono suddivisi gli strumenti sono: girodischi, molatori, armoniche, zampogni, radio, segopiatti e speciali, tutti comandati tramite Arduino ${ }^{54}$.

I girodischi consistono in dei giradischi di bassa qualità e auto-amplificati, con una connessione diretta tra il motore e la piastra rotante. Comandati con dei relè che controllano l'alimentazione dei motori, il risultato sonoro consiste nel movimento di rotazione della puntina su un disco (da scegliere liberamente dal repertorio orchestrale) a 33 giri suonato a 45 giri e azionato velocemente.

I molatori consistono in componenti recuperati da un mangiacassette smantellato, che, sfregando diverse superfici metalliche, generano un suono poco intenso e molto inarmonico.

Le armoniche consistono in tre armoniche a bocca fissate e messe in vibrazione da tre asciugacapelli. Il motore dell'asciugacapelli è isolato dal resistore di calore e viene alimentato corrente a $12 \mathrm{~V}$, e le armoniche a bocca devono essere intonate chiudendo i fori con del nastro adesivo.

Gli zampogni consistono in quattro set di tre flauti dolci connessi e messi in eccitazione da un asciugacapelli modificato, come quelli delle armoniche. I set di tre flauti sono:

${ }^{54}$ Un video dimostrativo sugli esperimenti condotti su questa orchestra residuale è reperibile all'indirizzo <https://vimeo.com/81482138> 
1. Tre flauti dolci soprano;

2. Due flauti dolci soprano e un sopranino;

3. Due flauti dolci soprano e un sopranino;

4. Un flauto dolce soprano e due sopranini.

Tutti i flauti hanno i fori chiusi in corrispondenza di determinate diteggiature predeterminate; il risultato timbrico sarà alterato microtonalmente per via della pressione discontinua e controllabile degli asciugacapelli.

Le radio consistono in tre radio e radiosveglie modificate in modo tale da avere un relè che apre o interrompe le connessioni con gli altoparlanti, ottenendo un suono di accendimento/spegnimento del segnale radio - impostato su una frequenza senza segnale. Le prime due radio ricevono un segnale AM, mentre la terza radio un segnale FM.

I segopiatti sono dei coltelli elettrici modificati che agiscono su dei piatti di batteria rotti.

Gli strumenti speciali sono quattro strumenti che non rientrano in nessuna delle famiglie formalizzate precedentemente, e sono lo Spremoagrume (uno spremiagrumi azionato elettronicamente, da un suono di rotazione scuro), il Rasoio (un rasoio elettrico posizionato in una scatola di metallo riempita parzialmente con bottoni di plastica, per ottenere un suono simile ad un buzz), e la Lampadina, che prevede il suono di attivazione del relè e provvede allo stesso tempo una componente visiva.

Gli strumenti sono quindi disposti circondando i musicisti, come in Fig. 11.

In Regnum Vegetabile gli strumenti automatizzati sono organizzati nelle famiglie trombi, zampogni, armoniche e speciali.

Tutte queste famiglie sono caratterizzate dall'utilizzo di un totale di 30 asciugacapelli modificati - similmente ai casi delle armoniche de degli zampogni di Regnum Animale, che qua vengono implementati.

In questo caso le armoniche sono sette, e la terza e la sesta hanno i cavi dell'asciugacapelli invertiti per far compiere un atto di aspirazione al mezzo eccitante. Gli zampogni invece in questo caso sono sette.

La famiglia dei trombi consiste in 10 tubi in PVC che funzionano come risuonatori per delle trombette giocattolo.

Gli strumenti speciali consistono in un ancetto (uno strumento ad ancia giocattolo), una fischietta (un fischietto giocattolo), due ocarine (costruite con due ocarine ciascuno), e due sirenetti (costruiti unendo insieme tre sirene giocattolo). A partire da Regnum Vegetabile, la partitura viene completata da un'appendice illustrativa sulla configurazione di SuperCollider e Arduino per il corretto funzionamento degli strumenti. Nel caso dei trenta asciugacapelli due Arduino controllano l'intero sistema.

Gli strumenti sono disposti come in Fig. 12 e 13.

In Regnum Lapideum le famiglie implementate sono coni, eolii, tole, sistri, cimbali, speciali e cetro.

Il cetro è il cetro basso già illustrato per Selva Petrosa. I due cordofoni automatizzati da Valle per il suo ciclo sono stati entrambi implementati per Regnum Lapideum, ma in questo caso è stato utilizzato solamente il cetro basso, controllato da Arduino. 


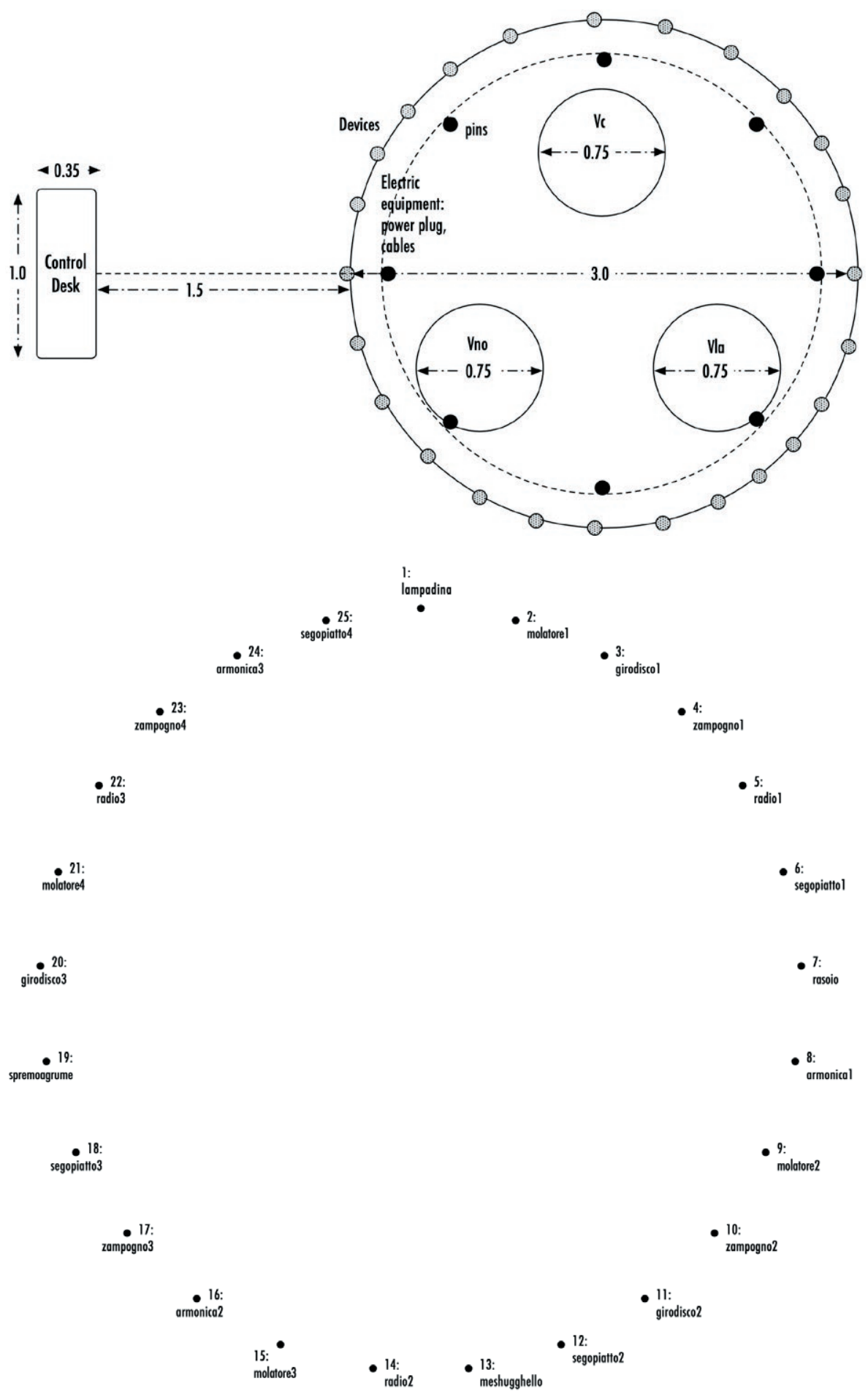

Figura 11. A. Valle, M. Lanza, Regnum Animale, inedito. Disposizione degli strumenti. 


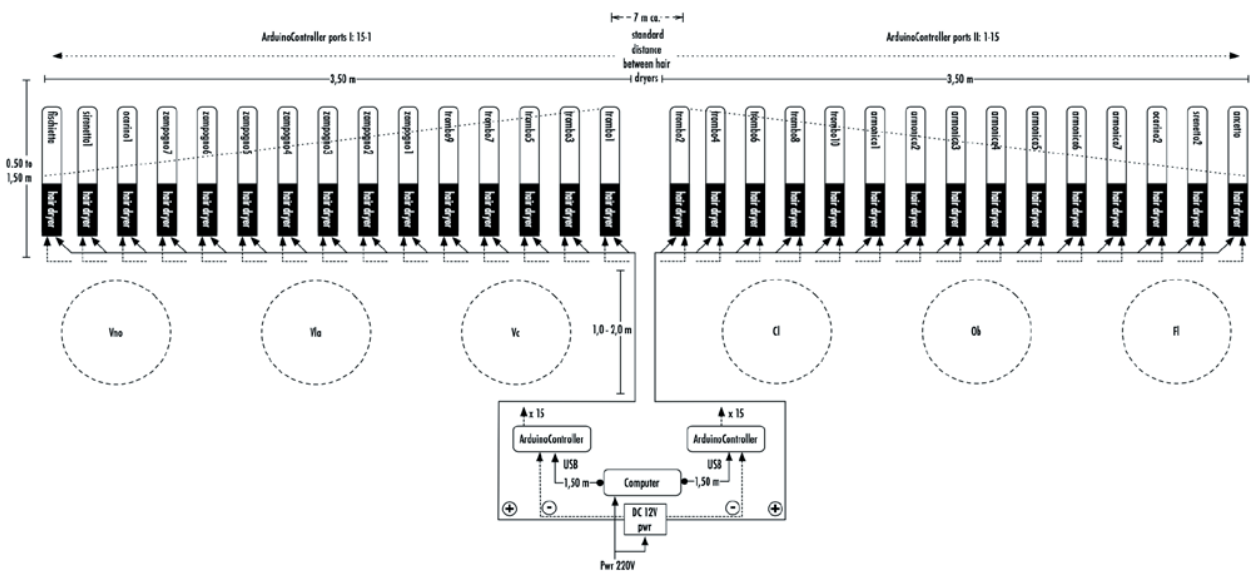

Figura 12. A. Valle, M. Lanza, Regnum Vegetabile, inedito 2014. Disposizione degli strumenti.

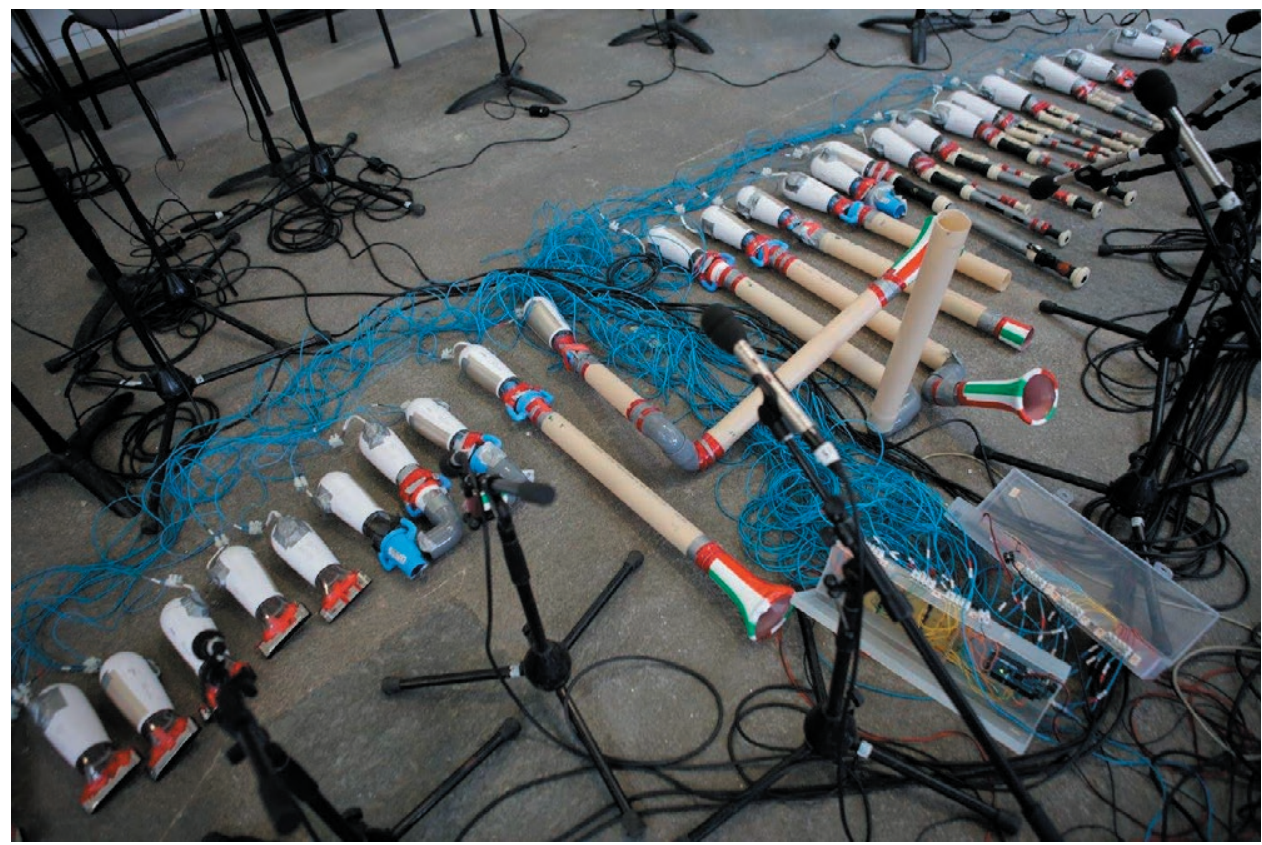

Figura 13. Disposizione degli strumenti per il Festival Les Amplitudes, 2018, CPablo Fernandez, Festival Les Amplitudes, 2018.

Gli eolii consistono in strumenti ottenuti con degli asciugacapelli da $24 \mathrm{~V}$ modificati, i quali mettono in eccitazione dei tubi in PVC connessi ad imboccature di flauti dolci, ed attivati da relè a $12 \mathrm{~V}$, controllati da Arduino.

Le tole consistono in sei strumenti metallici percussivi, ottenuti inserendo una barra di metallo all'interno di contenitori di metallo. L'automazione consiste in motorini rotante da $12 \mathrm{~V}$ che attuano frizione sulla barra di metallo, controllati da Arduino. 
I sistri e cimbali hanno lo stesso modo di attivazione: un attuatore rotante controllato da Arduino con dei campanellini che attua una frizione su un corpo metallico risonante. I primi (chiamati skipper e velvet) consistono in contenitori di tabacco da pipa circolari; i secondi (chiamati caldarroste e gianduja) sono contenitori più grandi con capacità risonanti differenti.

Gli speciali consistono in due strumenti chiamati cocalola e anciolio, entrambi controllati da Arduino. La cocalola consiste in una bottiglia di coca-cola in vetro, messa in risonanza con un asciugacapelli modificato da $12 \mathrm{~V}$. L'anciolio è un'ancia a basse frequenze dentro una scatola di metallo attivata da una ventola a $12 \mathrm{~V}$.

I coni infine sono gli unici strumenti ad essere controllati non da Arduino, bensì da una scheda audio con relativo amplificatore a 8 canali. I coni consistono in 8 altoparlanti woofer $(120 \mathrm{~W}, \varnothing 165 \mathrm{~mm})$, posizionati direttamente per terra e rivolti verso l'alto, con degli oggetti posti sopra, che funzionano come risonatori. Il segnale audio che riproducono gli speaker corrisponde ad una serie di impulsi, atti a mettere in risonanza gli oggetti, che funzionano appunto come risonatori e a loro volta indicati con nomi precisi, e sono organizzati in cinque sottofamiglie. La prima comprende i lingamMinor e lingamMaior, due tubi molto grandi in pvc, dal diametro di $16 \mathrm{~cm}$ per ottenere una risonanza bassa dell'impulso; la seconda comprende tre scatole di metallo di tre grandezze diverse che fungono da risonatori di metallo, chiamate in partitura panettone, biscotti e arancio. Ottone è il terzo risonatore, e consiste in una scatola rigida d'ottone che produce un suono sferragliante. Il quarto risonatore è il cappello, un assemblaggio di un cilindro di metallo posto sopra un suporto dello stesso materiale. L'ultimo, tibet, corrisponde ad una piastra di metallo sulla quale viene posta una piccola campana tibetana, per ottenere un suono simile all' $h i$-hat percosso.

La disposizione è illustrata in Fig. 14.

Per quanto riguarda la componente strumentale tradizionale, gli strumenti a corda sono preparati con del pattafix, con quantità da impiegare e in posizioni minuziosamente indicate in partitura, in modo tale da ottenere un suono complesso a partire dall'intervento fisico sui punti nodali di una corda semplice. Questa tecnica è usata dal compositore veneziano fin da La bataille de Caresme et de Charnage, (del 2012, per violoncello e pianoforte, esistente anche in una versione per violoncello ed elettronica) come frutto della collaborazione con la violoncellista e compositrice Séverine Ballon.

Le miniature che compongono i quattro brani di Systema Naturae sono secondo un principio decrescente con ordine 6 nei regna, come illustrato in Tavola 1.

La natura di "omaggi" delle singole miniature è messa in evidenza dai compositori stessi nel libretto del disco inciso da mdi ensemble, tra cui si ritrovano riferimenti alla storia della musica occidentale, alla storia della computer music, riferimenti rock e metal, riferimenti a campi artistici diversi da quello prettamente musicale, agli esecutori dei brani, e omaggi ad amici e persone vicine:

La seguente è una genealogia non esaustiva dei 60 autori in termini di omaggio/ispirazione: Séverine Ballon, Alighiero Boetti, Zdenek Burian, John Cage, Giorgio Casati, John Conway, Harry Dacre, Claude Debussy, The Dillinger Escape Plan, Umberto Eco, Helmet, Jimi Hendrix, John Holland, Emilio Isgrò, Stephen Jay Gould, Tom Johnson, 


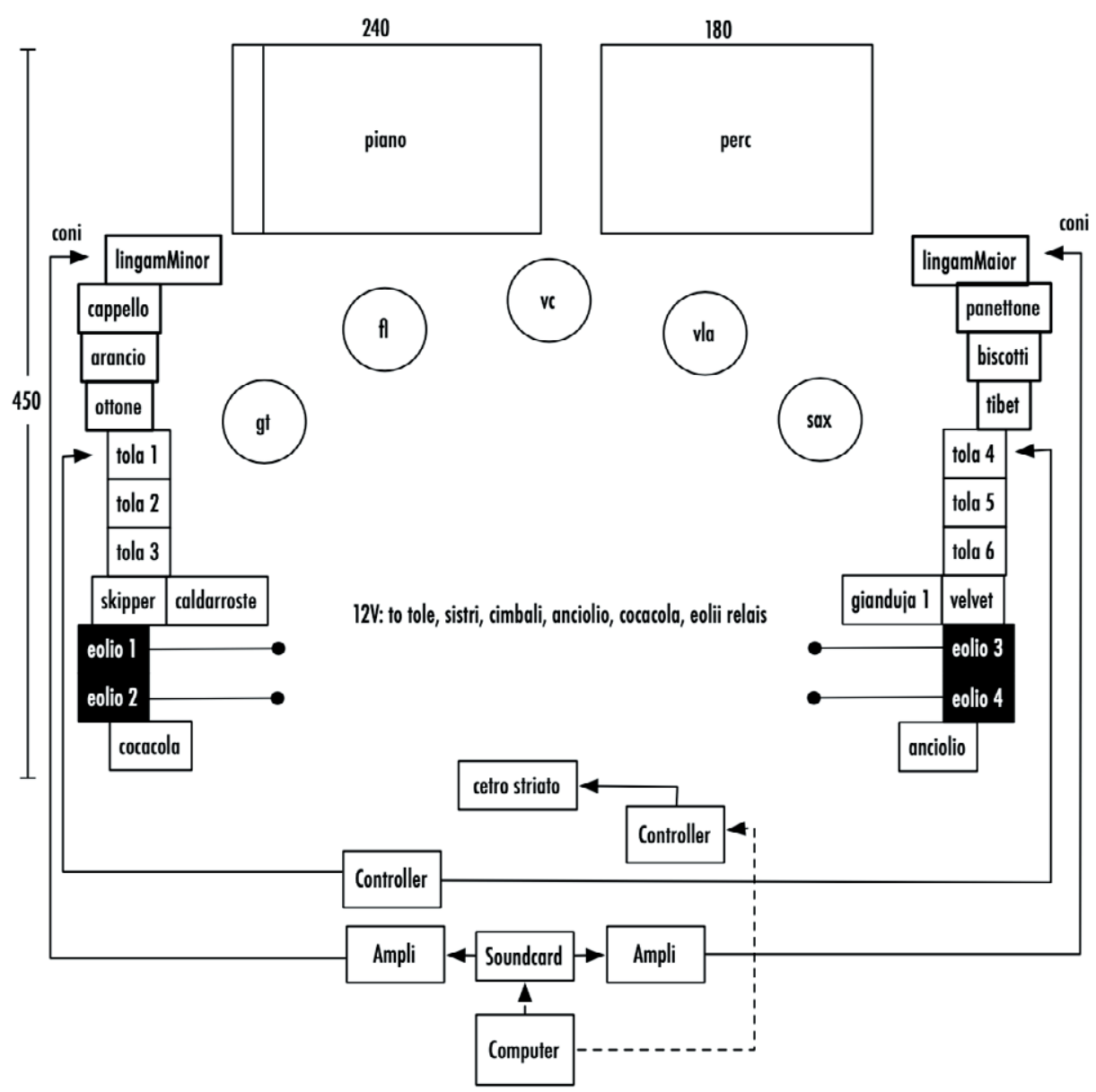

Figura 14. A. Valle, M. Lanza, Regnum Lapideum, inedito 2016. Disposizione degli strumenti.

Bettina Junge, György Ligeti, Aristid Lindenmayer, Hampus Lindwall, Carl Linnaeus, Max Mathews, Melvins, Meshuggah, Luigi Nono, Simone Pappalardo, Primus, David Raup, Steve Reich, Dario Sanfilippo, Toti Scialoja, Salvatore Sciarrino, Johann Sebastian Bach, gli uccelli di Ciriè, Gesualdo da Venosa, Anton Webern, Iannis Xenakis ${ }^{55}$.

Come indicato in Tavola 2, le miniature che compongono Sistema Naturae nell'incisione di mdi ensemble sono state talvolta cambiate di posizione all'interno della macrostruttura di Regnum Animale, aggiungendo inoltre Furusthmachibichnia, quarto numero di Fossilia, e omaggio a Future Breed Machine, brano degli svedesi Meshuggah.

55 A. Valle, M. Lanza, Systema Naturae, booklet cd mdi ensemble, Stradivarius 2019. 


\begin{tabular}{|l|l|l|l|}
\hline Regnum Animale & Regnum Vegetabile & Regnum Lapideum & Fossilia \\
\hline Minaeptacta gringi & Nononophis janeziarii & Aligurius & Hinicinichnia \\
\hline Phola reicha & Uelerinea ballus & Gagalida & Seuschylichnia \\
\hline Taleus photothodecae & Tocactocepia eventaeticans & Echelechelena & Aranichnia \\
\hline Zampychis flalutengla & Hipseus lanthicus & Matestontes & Furusthmachibichnia \\
\hline Cteromelis udivetusi & Schinia groumbusia & Anionidia & Totalatelontelmichnia \\
\hline Graphas lopongens & Ferocylopia erossini & Eliteralates & Sectessichnia \\
\hline Sectiditomys stonisius & Ismiosia papanabuis & Elenion & \\
\hline Urysilomys hyssii & Ariolactus usteginsiphillemena & Chrisopiris & \\
\hline Omysomyomys cacaca & Hodolindereus hyboalga & Iactopia & \\
\hline Ioris casachocii & Erelmatus clens & Caracon & \\
\hline Zamonicomus monica & Canochia usiva & Gerillidon & \\
\hline Adius geradii & Disia belga & \\
\hline Cteromelis melins & Hipseus valos & & \\
\hline Nomotaus yansicomolis & Reocerantroma phenaudi & & \\
\hline Vinteroicis intermans & Chylicerela eucucta & & \\
\hline Feriocetus petrii & Melonthora cirencesus & & \\
\hline Durophos wienocia & Bindronocereus ligenatos & & \\
\hline Cistomalpha notus & Pentochtelacinia xissisiis & & \\
\hline Acaprimomyda tibie & & & \\
\hline Onomys vallorueusca & & & \\
\hline Urophoturonta glistripus & & & \\
\hline Daripessus yantillippicus & & & \\
\hline Urochronopus stoniarens & & & \\
\hline Hoopus lindens & & & \\
\hline
\end{tabular}

Tavola 1. Ordine delle miniature che compongono Systema Naturae, come presentate nel disco Systema Naturae, mdi ensemble, Stradivarius 2019.

\begin{tabular}{|l|l|l|l|}
\hline Regnum Animale & Regnum Vegetabile & Regnum Lapideum & Fossilia \\
\hline Minaeptacta gringi & Nononophis janeziarii & Aligurius & Hinicinichnia \\
\hline Phola reicha & Uelerinea ballus & Gagalida & Aranichnia \\
\hline Taleus photothodecae & Tocactocepia eventaeticans & Echelechelena & Seuschylichnia \\
\hline Zampychis flalutengla & Hipseus lanthicus & Matestontes & Totalatelontelmichnia \\
\hline Cteromelis udivetusi & Schinia groumbusia & Anionidia & \\
\hline Graphas lopongens & Ferocylopia erossini & Eliteralates & \\
\hline Sectiditomys stonisius & Ismiosia papanabuis & Elenion & \\
\hline Urysilomys hyssii & Ariolactus usteginsiphillemena & Chrisopiris & \\
\hline Omysomyomys cacaca & Hodolindereus hyboalga & Iactopia & \\
\hline Pteronulephis urachotrons & Erelmatus clens & Caracon & \\
\hline Ioris casachocii & Canochia usiva & Gerillidon & \\
\hline Zamonicomus monica & Disia belga & \\
\hline Nomotaus yansicomolis & Hipseus valos & & \\
\hline Adius geradii & Reocerantroma phenaudi & & \\
\hline Cteromelis melins & Chylicerela eucucta & & \\
\hline Onomys ucetasolanzondaroma & Melonthora cirencesus & & \\
\hline Vinteroicis intermans & Bindronocereus ligenatos & & \\
\hline Hoopus lindens & Pentochtelacinia xissisiis & & \\
\hline Durophos wienocia & & & \\
\hline Cistomalpha notus & & & \\
\hline Atottotis melitopuma & & \\
\hline Wiluscomylanycanonis bacus & & & \\
\hline Acaprimomyda tibie & & & \\
\hline Onomys vallorueusca & & & \\
\hline Urophoturonta glistripus & & & \\
\hline Daripessus yantillippicus & & & \\
\hline Urochronopus stoniarens & & & \\
\hline Feriocetus petrii & & \\
\hline
\end{tabular}

Tavola 2. Ordine delle miniature che compongono Systema Naturae, come presentate nelle partiture inedite di Regnum Animale (2013), Regnum Vegetabile (2014), Regnum Lapideum (2016), Fossilia (2017).

Si entrerà quindi nel dettaglio delle tecniche compositive algoritmiche sviluppate negli esempi tratti da Regnum Vegetabile (Ulerinea ballus, Isimiosia papanabuis, Disia Belga), e Fossilia (Totalatelontelomichnia) 


\subsubsection{Ulerinea ballus}

Ulerinea ballus (Fig. 15 e 16) è la seconda miniatura di Regnum Animale, e rappresenta uno dei casi chiave dell'applicazione di recenti tecniche compositive algoritmiche di Mauro Lanza.

La maggior parte delle tecniche sviluppate da Lanza per Systema Naturae consistono in diversi sistemi di targeting di un elemento rispetto a diversi database di dati simbolici e notazionali.

Per Ulerinea ballus Lanza impiega una tecnica di individuazione automatica di gradi di prossimità spettrale tra uno spettro $\mathrm{A}$ e uno spettro $\mathrm{B}$, generando una serie di ulteriori spettri i cui valori sono equidistanti tra A e B. Questa tecnica viene impiegata per la prima volta da Mauro Lanza in Ludus de Morte Regis, brano del 2013 per coro ed elettronica, e la illustra nella lezione tenuta per il cursus dell'IRCAM dello stesso anno ${ }^{56}$.

In video 2, il compositore illustra l'applicazione di questo sistema nel caso di Ulerinea ballus.

[Video 2 https://zenodo.org/record/4903388]

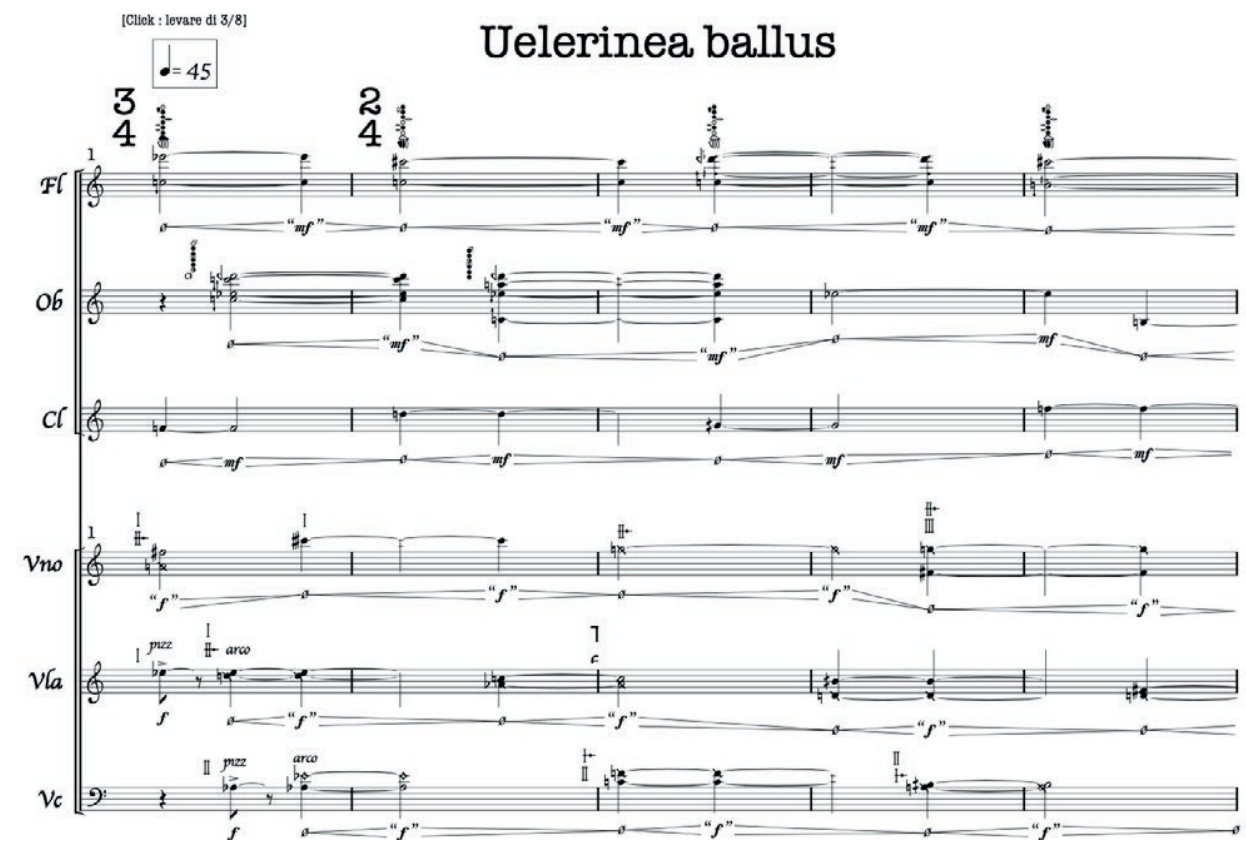

Figura 15. A. Valle, M. Lanza, Regnum Vegetabile, inedito 2014. Ulerinea ballus, partitura strumentale.

$56<$ https://medias.ircam.fr/embed/media/xd098bc_the-making-of-ludus-de-morte-regis-vf> 


\section{II: Uelerinea ballus}

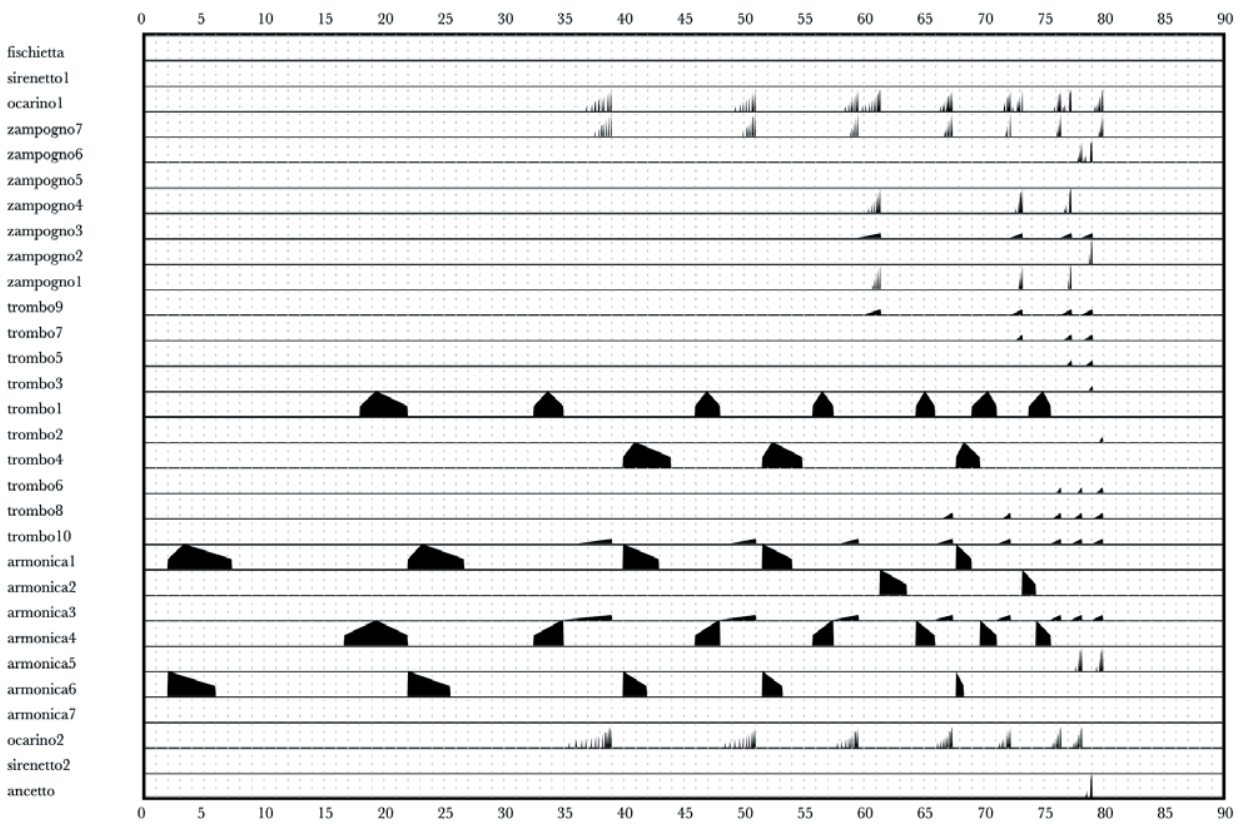

Figura 16. A. Valle, M. Lanza, Regnum Vegetabile, inedito 2014. Ulerinea ballus, partitura degli strumenti elettromeccanici.

\subsubsection{Isimiosia papanabuis}

Il brano Isimiosia papanabuis (Fig. 17 e 18) è il settimo brano di Regnum Vegetabile, omaggio a Simone Pappalardo.

La tecnica compositiva algoritmica qui sviluppata consiste nell'audioparismo, introdotto nel paragrafo 4.2.

Partendo dai termini utilizzati in zoologia per distinguere le modalità di generazione - vista in senso chomskiano - della specie (viviparità, oviparità, ovoviviparità), Valle nota come questa distinzione tassonomica prescinda dalle classi di esseri viventi, mettendo in luce le modalità di generazione della vita. Applicando questo assunto in musica, Valle chiamerà quindi "audioparità" una modalità di composizione in cui il dato musicale origina dal suono. Di conseguenza l'audioparismo descrive una modalità estetica e pratica fondata sull'audioparità.

Gli esempi di audioparismo qua riportati vedono la traslazione di microstrutture, in particolare al livello del singolo campione, in strutture temporalmente estese al livello dell'evento musicale, chiamando questa tecnica compositiva CampComp (SampComp in inglese). Nel caso qui illustrato vengono applicate due operazioni digitali al segnale sorgente del processo di conversione in campioni: il ricampionamento e la riquantizzazione. La prima prende per assunto la possibilità di manipolare il tasso di descrizione digitale di un segnale nel tempo, permettendone il ricampionamento ad un tasso di informazioni uguale o inferiore. La seconda, la riquantizzazione, definisce l'escursione 
entro cui le ampiezze del segnale sono ricondotte ad altezze. Le due operazioni introducono una perdita di informazione a livello strutturale e di fatto una componente di "errore", essendo operazioni non lineari.

Ma nell'esplorazione dell'errore consiste larga parte dell'interesse dell'operazione. Nelle parole del compositore:

\begin{abstract}
$\mathrm{Al}$ di là dello studio teorico, il caso più semplice consiste nel ricampionare prendendo un campione ogni n. Il nuovo tasso di campionamento srn sarà un sottomultiplo intero del campionamento originale sr, ovvero il periodo di campionamento sTn sarà un multiplo intero di sT. Per quanto riguarda la quantizzazione, si può definire una funzione di proiezione che associ per interpolazione lineare il valore nel dominio del campione $( \pm 1.0)$ a un valore in quello dell'altezza, approssimato per intero $( \pm 12)$. [...] Pur nella semplicità dell'esempio, due cose sembrano interessanti: i) la forma del segnale in qualche misura permane come forma della melodia, nel nuovo segnale mld; ii) la quantizzazione all'ottava temperata introduce una distorsione che si traduce in un insieme di terrazzamenti, per così dire ${ }^{57}$.
\end{abstract}

Questo approccio generativo della forma musicale secondo la conversione della più piccola unità di computazione del segnale audio in evento di articolazione rappresenta una conseguenza della visione della forma musicale per il compositore piemontese:

[...] quando uno ha la sfortuna come me di essere afflitto da una sindrome semiologica, non può esimersi dal considerare la dimensione simbolica delle forme, cioè il semplice fatto che una forma è un oggetto cognitivo, una sorta di invariante che può essere soggetta a processi di trasduzione, di conversione, variamente non lineare, come in una distorsione, eppure mantenere una sorta di salienza strutturale ${ }^{58}$.

Isimiosia papanabuis rappresenta un esempio di questa applicazione del processo di conversione di dati come generatore di forme musicali. Andrea Valle, infatti estrae l'impianto formale e articolativo con la tecnica dell'audioparismo da un campione audio di un secondo, tratto da una registrazione di un'improvvisazione del duo Otosimbionte, progetto musicale di Valle e Pappalardo. Nel Video 3, Andrea Valle esemplifica l'applicazione dell'audioparismo nel caso specifico del brano in analisi.

[Video 3 https://zenodo.org/record/4903408]

\title{
4.3.3 Disia belga
}

Il modello compositivo di Disia belga (Fig. 19 e 20), dodicesima miniatura di Regnum Vegetabile, è Daisy Bell, canzone popolare americana di fine XIX secolo. Daisy

57 A. Valle, SampComp: sample-based techniques for algorithmic composition, in Proceedings of the 22nd CIM, Udine 2018, pp. 128-135.

$58<$ https://www.musicaelettronica.it/campcomp-lineamenti-di-musica-audiopara-1/> 


\section{Ismiosia papanabuis}

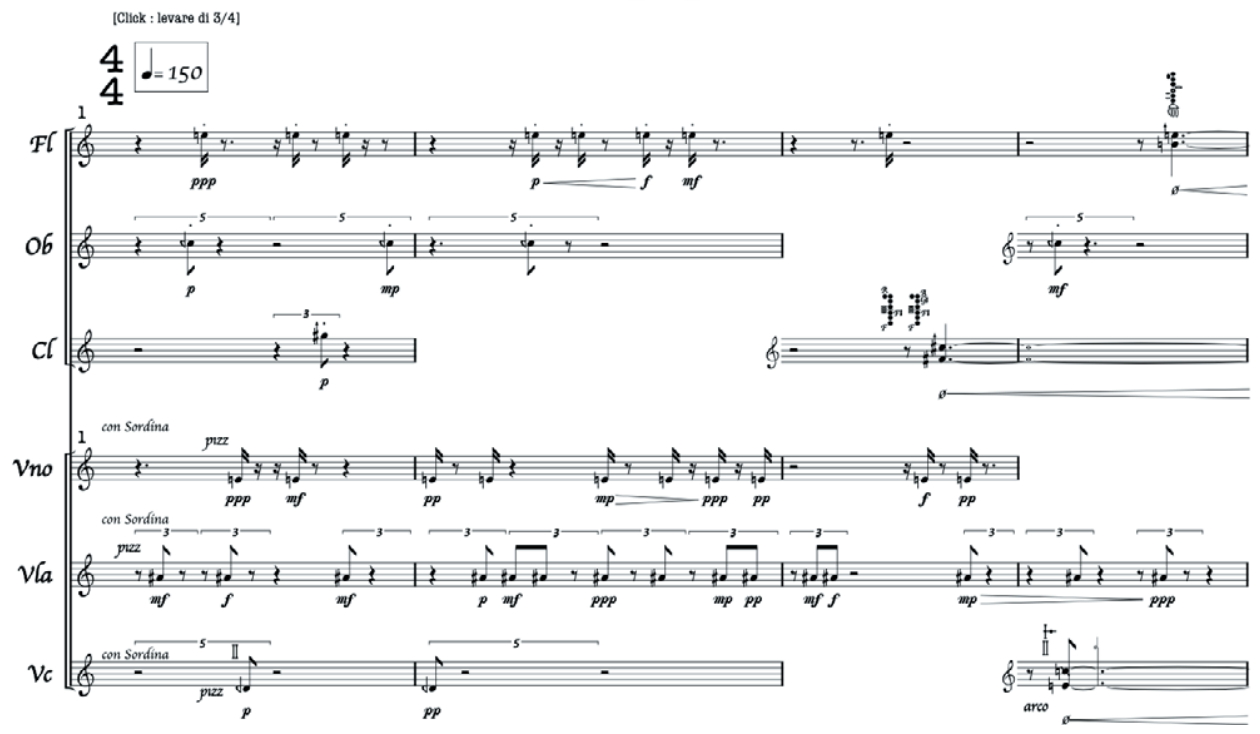

Figura 17. A. Valle, M. Lanza, Regnum Vegetabile, inedito 2014. Isimiosia papanabuis, partitura strumentale.

\section{VII: Ismiosia papanabuis}

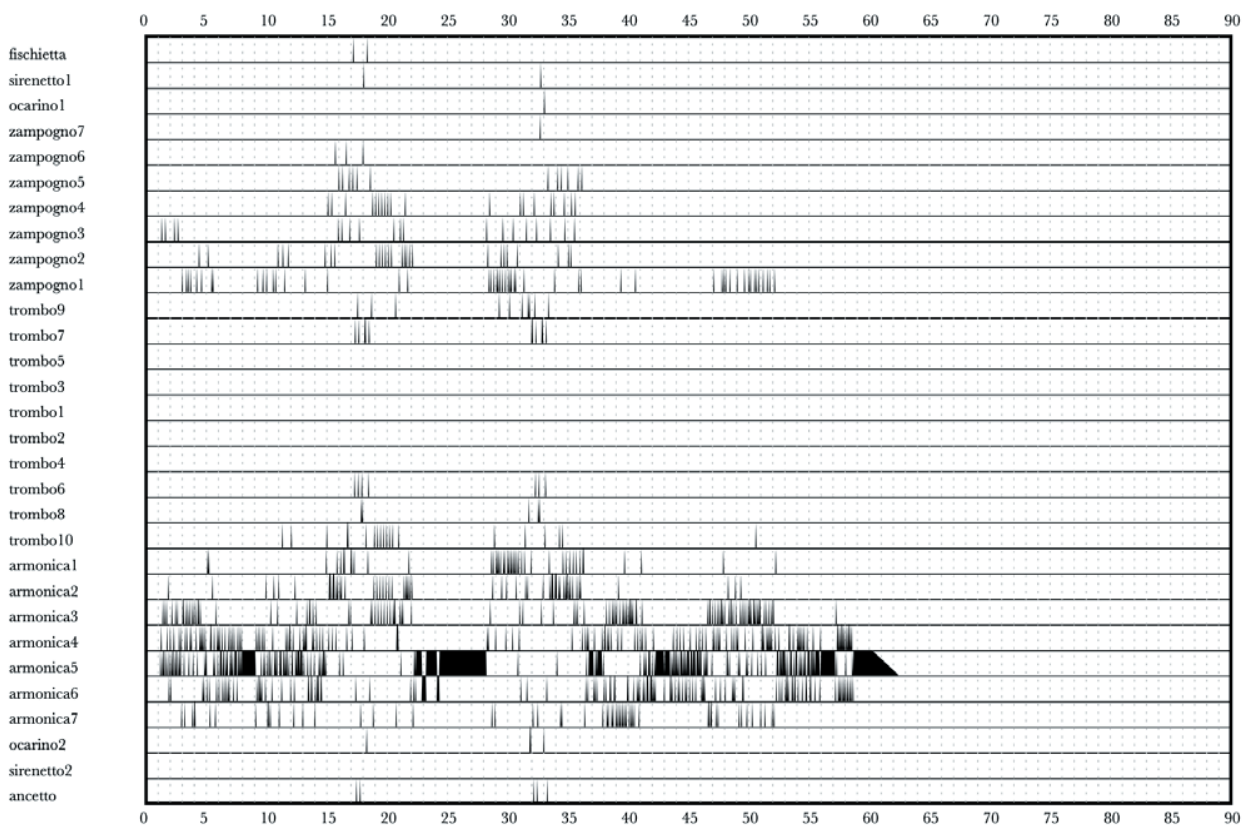

Figura 18. A. Valle, M. Lanza, Regnum Vegetabile, inedito 2014. Isimiosia papanabuis, partitura degli strumenti elettromeccanici. 

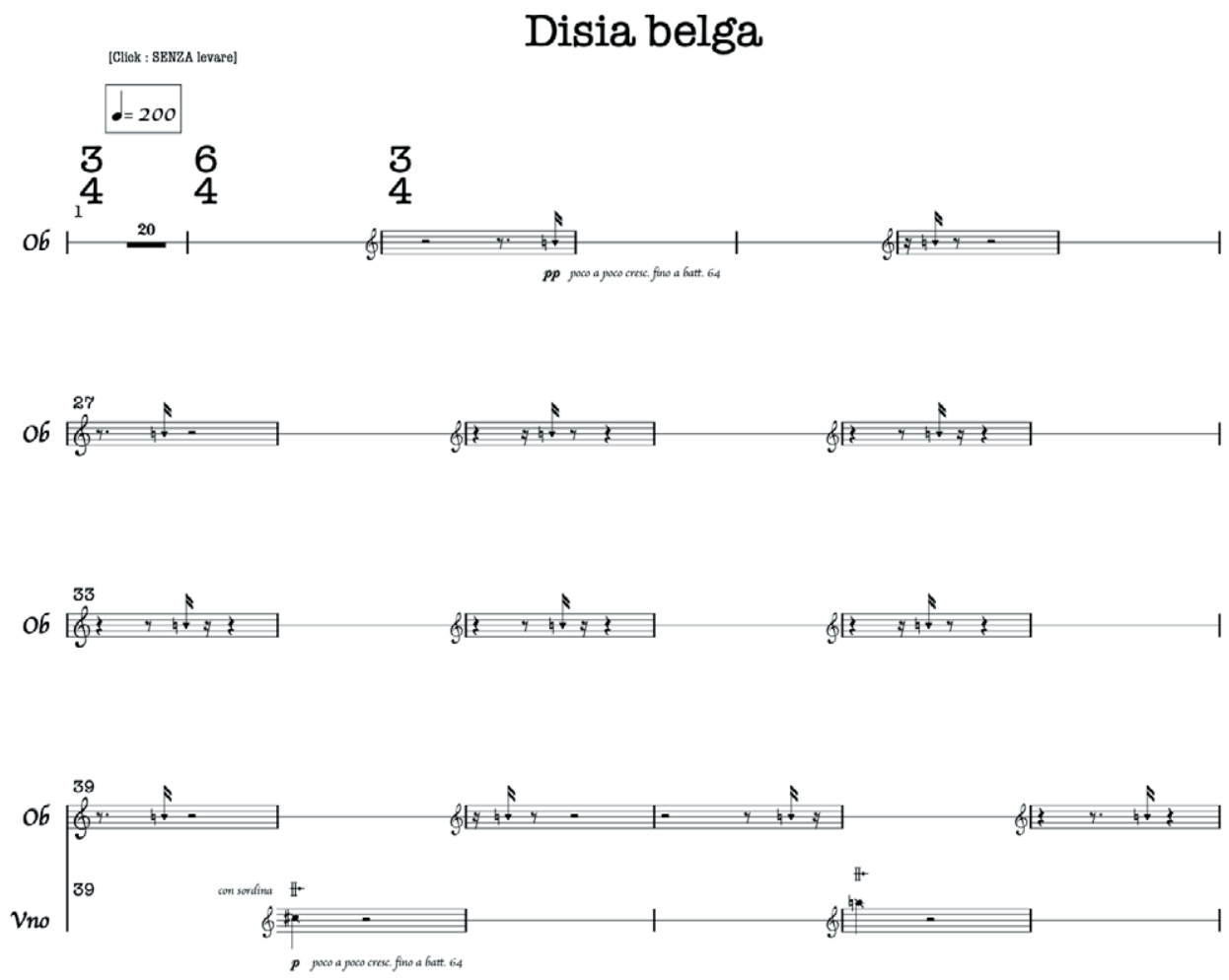

Figura 19. A. Valle, M. Lanza, Regnum Vegetabile, inedito 2014. Disia Belga, partitura strumentale.

Bell è inoltre uno dei primi brani nel 1961 in cui viene sperimentato il primo modello di speech synthesis implementato da Max Mathews all'interno dei Laboratori Bell, insieme alla più nota Bycicle Built for Two ${ }^{59}$. Il modello influenza l'organizzazione dell'articolazione e la caratterizzazione delle funzioni nell'orchestrazione che gli strumenti - acustici ed elettromeccanici - acquisiscono.

L'algoritmo sviluppato da Mauro Lanza consiste in un sistema di targeting tra l'input notazionale (Daisy Bell) e il database di analisi spettrale dei campioni audio registrati da Andrea Valle. Questo sistema comporta l'implemento di algoritmi di analisi del suono e di astrazione simbolica del dato analitico. Per fare questo, Lanza nei suoi algoritmi ha implementato all'interno di OpenMusic l'impiego dei kernel super vp e $p m 2$ del software di analisi Audioscuplt sviluppato all'IRCAM ${ }^{60}$. L'impiego in particolare di $p m 2$ consente a Lanza di computare automaticamente delle chord-seq analysis (un formato molto di astrazione in notazione tradizionale, in forma di accordi, del contenuto spettrale di un suono, possibile in Audiosculpt) dei campioni dei suoni pre-

59 N. Collins, M. Schedel, e S. Wilson, (a cura di), Electronic Music, Cambridge Introductions to Music, Cambridge University Press, Cambridge 2013.

$60<$ http://support.ircam.fr/docs/AudioSculpt/3.0/co/AudioSculptguideWeb.html> 
XII: Disia belga

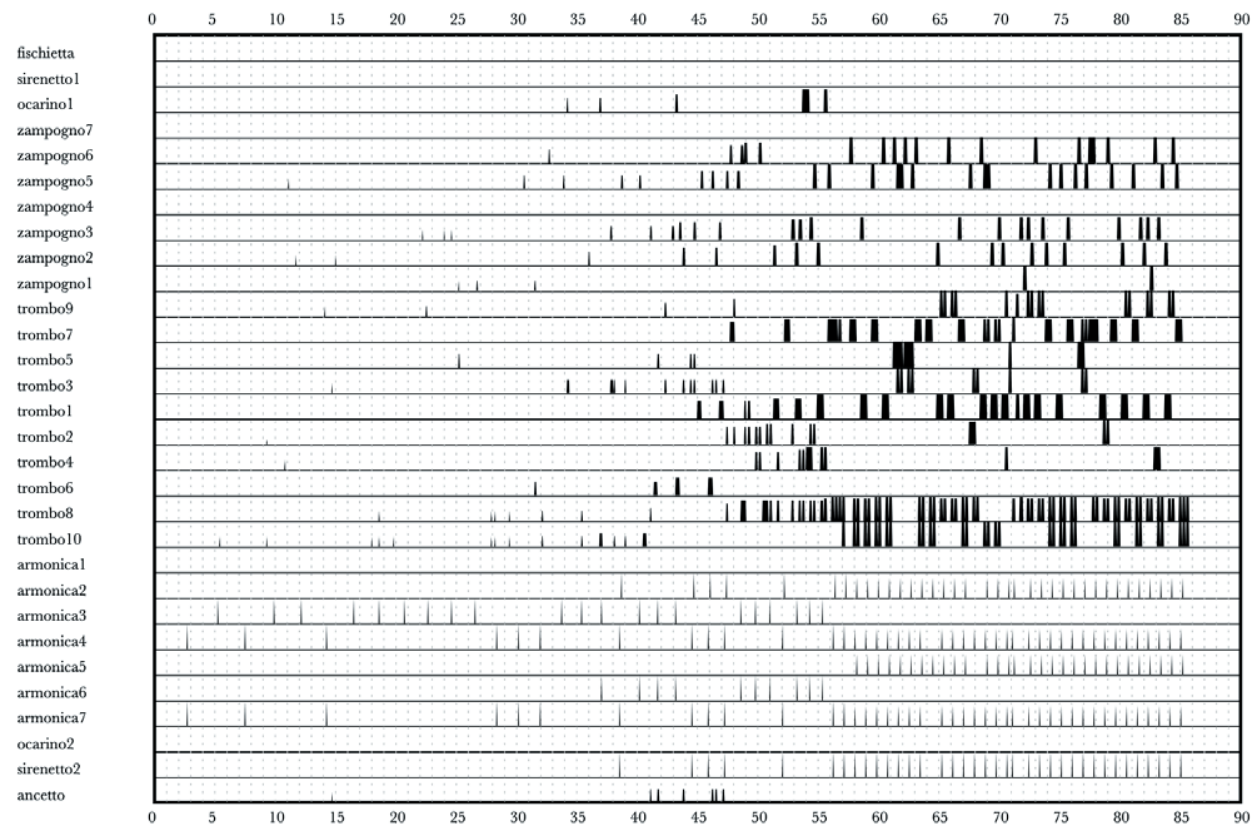

Figura 20. A. Valle, M. Lanza, Regnum Vegetabile, inedito 2014. Disia Belga, partitura degli strumenti elettromeccanici.

si in considerazione, confrontando automaticamente questo risultato con i database analitici, i cui dati simbolici sono ottenuti nella stessa maniera.

Nel Video 4, Mauro Lanza dimostra il complesso meccanismo di queste funzioni.

[Video 4 https://zenodo.org/record/4903412]

\subsubsection{Totalatelontelomichina}

Totalatelontelomichina (Fig. 21 e 22) è pensato come il brano conclusivo dell'intero ciclo - formalmente concluso con una breve coda. Nelle parole di Mauro Lanza, è un brano che "parla della morte», quindi la riduzione ultima allo stato di fossile. Il modello di riferimento è infatti la cantata sacra di Johann Sebastian Bach Liebster Gott, wann werd ich sterben? catalogata BWV 8, il cui testo tratta esplicitamente del tema della morte. Operativamente i compositori hanno segmentato algoritmicamente le simulazioni audio della gran parte dei brani del ciclo, considerando la battuta come unità di segmentazione, e creando un database con le analisi spettrali in forma simbolica di ogni singolo segmento. Questo database a sua volta è stato analizzato creando relazioni di prossimità tra i dati simbolici di ogni segmento. Questo processo ha dato alla luce tre grafici tassonomici dell'intero Systema Naturae, che, pur non essendo utilizzato a livello compositivo, sono utili per rappresentare visivamente la grande quantità di dati che i compositori sono andati a trattare algoritmicamente (Fig. 23). 


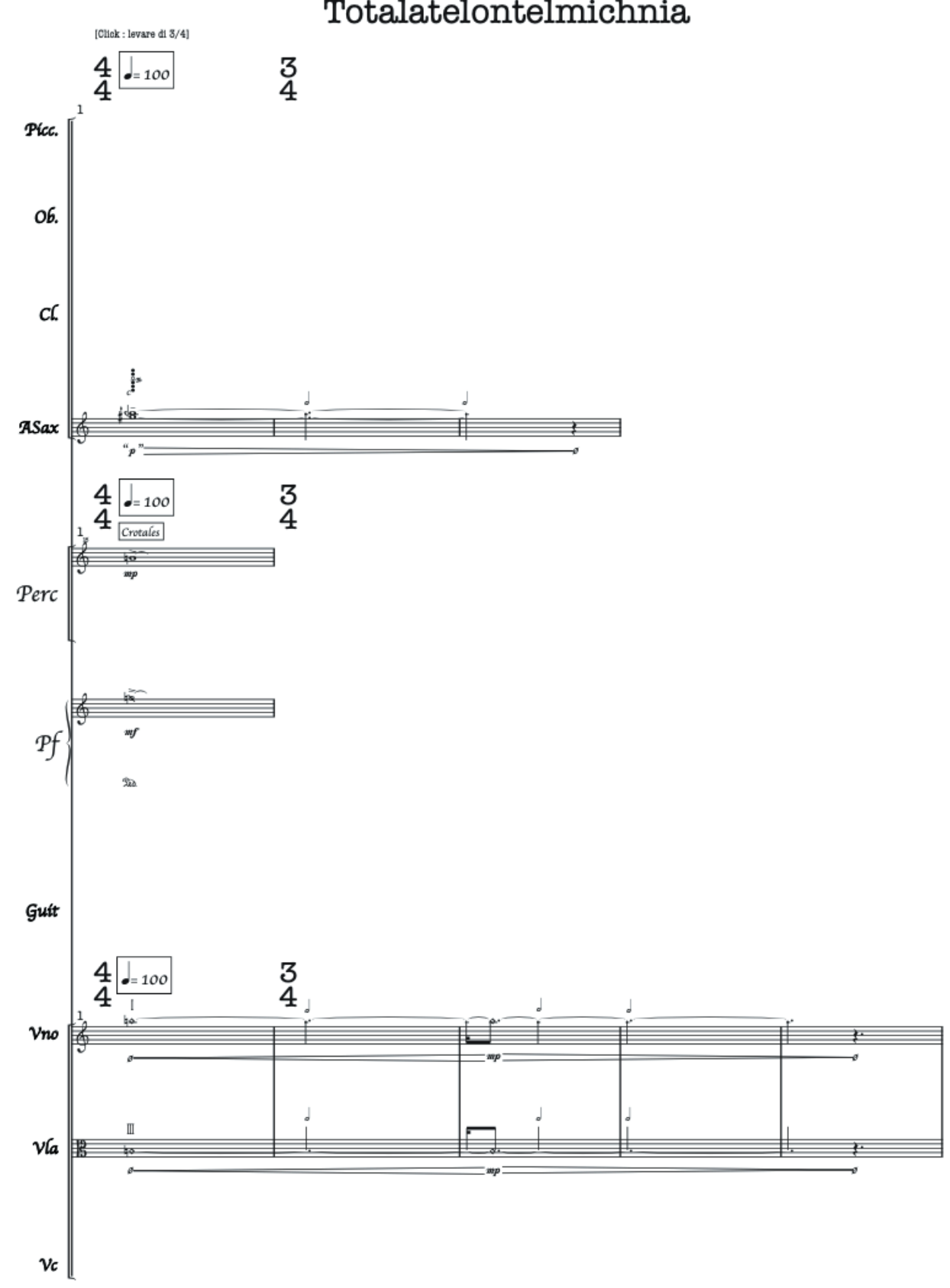

Figura 21. A. Valle, M. Lanza, Fossilia, inedito 2017. Totalatelontelomichina, partitura strumentale.

A livello operativo, prendendo il brano di Bach come modello simbolico di partenza, è stato effettuato il targeting del modello sul database creato dai compositori.

In Video 5 Mauro Lanza ne esemplifica l’applicazione.

[Video 5 https://zenodo.org/record/4903417] 


\section{IV: Totalatelontelmichnia}
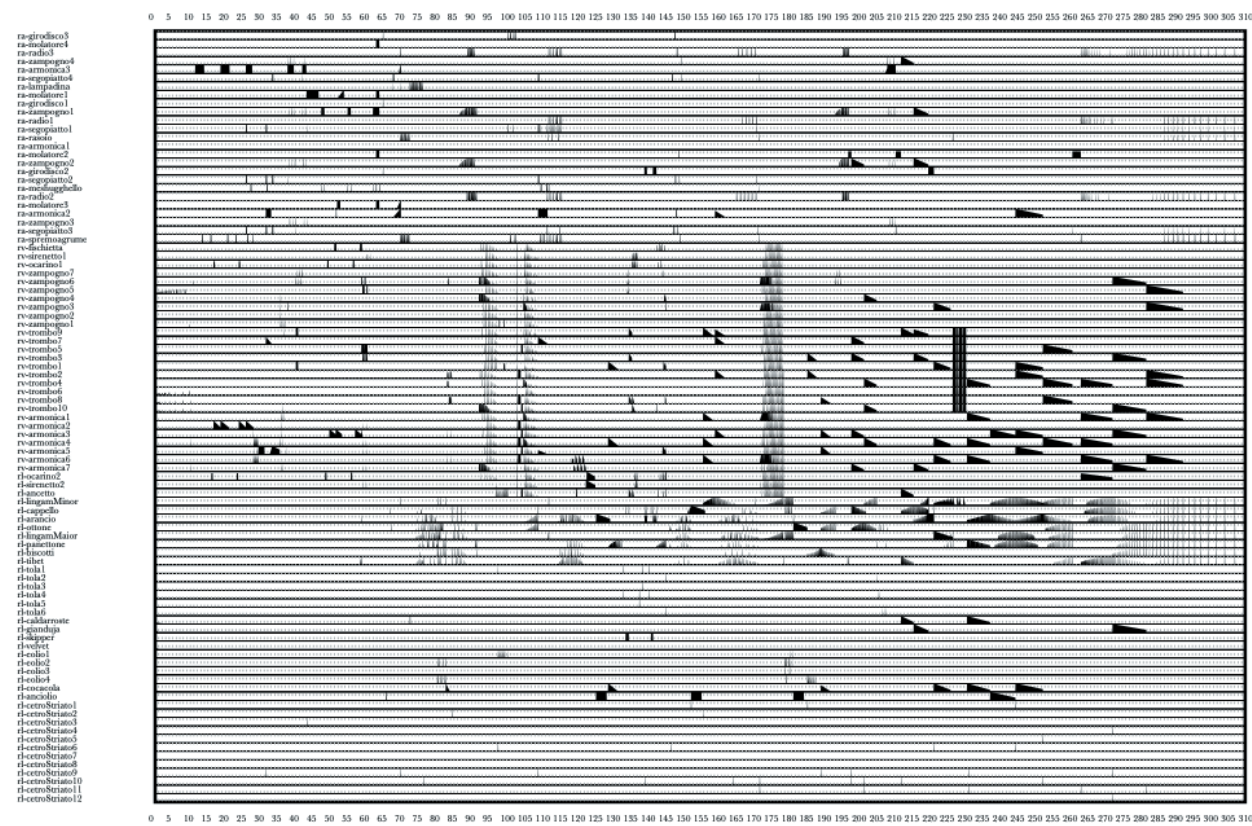

Figura 22. A. Valle, M. Lanza, Fossilia, inedito 2017. Totalatelontelomichina, partitura degli strumenti elettromeccanici.

\section{Simone Pappalardo: tra elettromagnetismo e automazioni}

Simone Pappalardo è compositore, performer e musicista elettronico, formatosi al Conservatorio Santa Cecilia di Roma, ha insegnato musica elettronica e informatica musicale nei Conservatori di Perugia, Bari, Latina, L'Aquila, Alessandria, e Sound Design alla RUFA Rome University of Fine Arts di Roma, è attivo principalmente in ambiti installativi e di creazione di strumenti di nuova liuteria elettromeccanica ed elettroacustica. La sua ricerca artistica infatti si radicalizza nell'interesse verso la creazione di sistemi artistici adattivi, autocontrollati e che agiscono con principi di feedback ricorsivi. Ha collaborato con Mauro Lanza per la costruzione degli strumenti per il brano The Kempelen Machine, e collabora con Andrea Valle nel già citato duo Otosimbionte e in pubblicazioni scientifiche.

Tra le attività di Pappalardo figura quella del restauro e del mantenimento delle sculture sonore di Mario Bertoncini, su commissione di Valeska Bertoncini per il Fondo Mario Bertoncini, di recente acquisizione della Fondazione Isabella Scelsi di Roma. Pappalardo sembra infatti condividere proprio con Mario Bertoncini - a cui dedica la prima opera per violino aumentato ${ }^{61}$ - alcune considerazioni teoriche che si riversano nella pratica musicale. In Per una bottega d'Arte dei nostri giorni. Istituzione

$61<$ https://vimeo.com/37180989> 


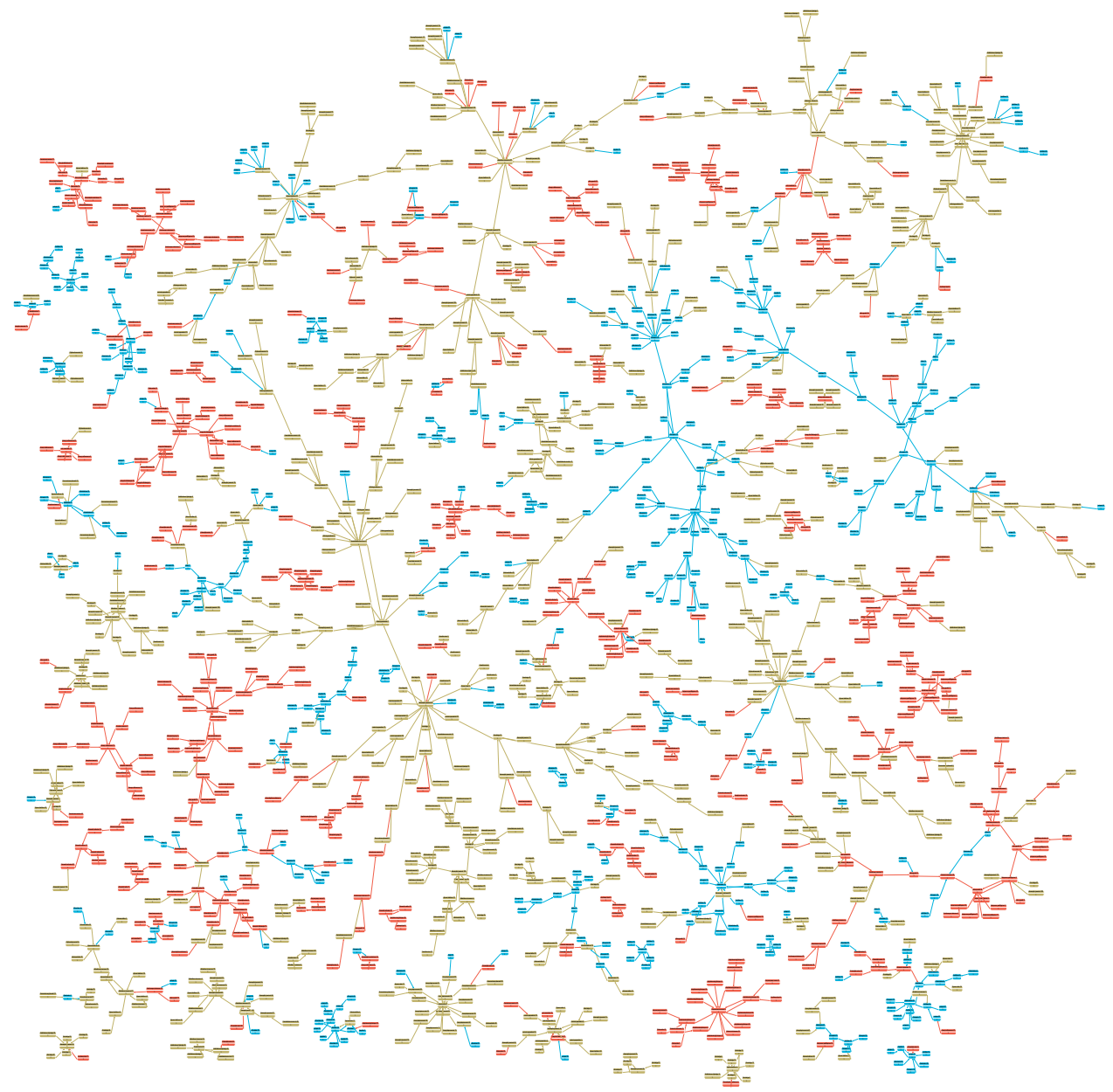

Figura 23. A. Valle, M. Lanza, diagramma di prossimità dei contenuti spettrali delle singole battute della quasi totalità del ciclo Systema Naturae.

di un centro sperimentale di ricerca sul suono, considerato in funzione delle esigenze compositive più avanzate, e sull'interazione tra fenomeni acustici, ottici e gestuali, Bertoncini ragiona sugli aspetti teorici e di ricerca intorno alla creazione di un apparato strumentale:

Colui che agisce, l'artista [...] [dovrebbe] organizzare con assoluta liberà i propri strumenti, ovvero i materiali necessari alla realizzazione dell'oggetto e di dominarne nei minimi particolari le tecniche relative. Tali materiali - siano essi strumenti acustici o elettronici, la differenza non è molta - sono sistemi chiusi ed esercitano, sulla fantasia dell'artista un'analoga costrizione ${ }^{62}$.

62 Mario Bertoncini, Per una bottega d'Arte dei nostri giorni. Istituzione di un centro sperimentale di ricerca sul suono, considerato in funzione delle esigenze compositive più avanzate, e sull'interazione tra fenomeni 
La composizione quindi, sia per Bertoncini che per Pappalardo, consiste nell'esplorare le qualità proprie del sistema chiuso, determinate parametricamente con finalità compositive. Secondo Pappalardo infatti:

La forma [...] si adatta al materiale - cioè agli strumenti e agli spazi - di cui è composta; con le tecnologie questo processo, attuato precedentemente in modo non sempre consapevole, diviene estremamente e facilmente controllabile, se ne può quindi ridefinire la centralità nella prassi compositiva. E questa è una grande novità, mettere al centro della composizione il materiale di cui è composta cioè lo strumento o l'algoritmo o il timbro $\mathrm{o}$ ancora il gesto sonoro. Ma con la tecnologia possiamo anche andare in senso inverso a questo processo. Possiamo cioè ridefinire completamente spazio e materiali in modo che ridisegnino la forma della nostra composizione ${ }^{63}$.

Mentre in Bertoncini il modello strumentale elettronico viene criticato assumendo maggiore complessità nell'organizzazione delle modalità organizzative e esecutive, per Pappalardo diventa terreno fertile di ricerca, attraverso la sperimentazione di tecniche con campi elettromagnetici modulati, sistemi di physical computing, algoritmi adattivi, e tecniche di feedback acustico e di dati computazionali. Come per Bertoncini, al centro del suo interesse musicale c'è l'idea di gesto musicale performativo:

The idea of building instruments comes from the theatre. From that experience I derived a strong interest towards physical gestures. Even sound could be seen as the memory of an action. There is no sound which has not been produced by a gesture. I therefore find it interesting to rethink classical instruments - or to develop new ones in order to investigate this relationship between physical gesture and sound in different ways, by minimizing or maximizing it and testing its limits ${ }^{64}$.

Gli strumenti così realizzati, che emancipano il prodotto sonoro dal gesto umano, abbracciano la definizione di orchestra residuale data nel primo paragrafo, dando nuovo valore acustico a oggetti con una destinazione di mercato estranea al mondo musicale, o rivalutando le possibilità storicamente imposte degli strumenti classici. Il complesso parco strumentale concepito da Pappalardo viene controllato o attuato con diverse tecniche, in particolare di physical computing e di elettromagnetismo; verranno qui illustrati esempi tratti dalle opere Orchestra Fragile, Forte e Millis().

Orchestra Fragile consiste in un'opera installativa per il Media Art Festival del 2017, nata dalla residenza artistica presso il Goethe-Institut di Berlino, e dedicata alla città tedesca. L'installazione consiste nello studio approfondito del concetto di fragilità dei materiali strumentali utilizzati: vasi e recipienti di vetro di diverse dimensioni, acquistati da artigiani e vetrai berlinesi, messi in attuazione con la tecnica della modulazione di campi elettromagnetici, costruiti come nello schema di Fig. 24.

acustici, ottici e gestuali, in «La bottega del suono. Mario Bertoncini. Maestri e allievi», C. Mallozzi, D. Tortora (a cura di), Editoriale Scientifica, Napoli 2017, pp. 123-127.

$63<$ https://www.ilsaxofonoitaliano.it/artisti/pappalardo-simone/>

64 <https://www.fluid-radio.co.uk/2013/05/postcards-from-italy-rome-simone-pappalardo/> 


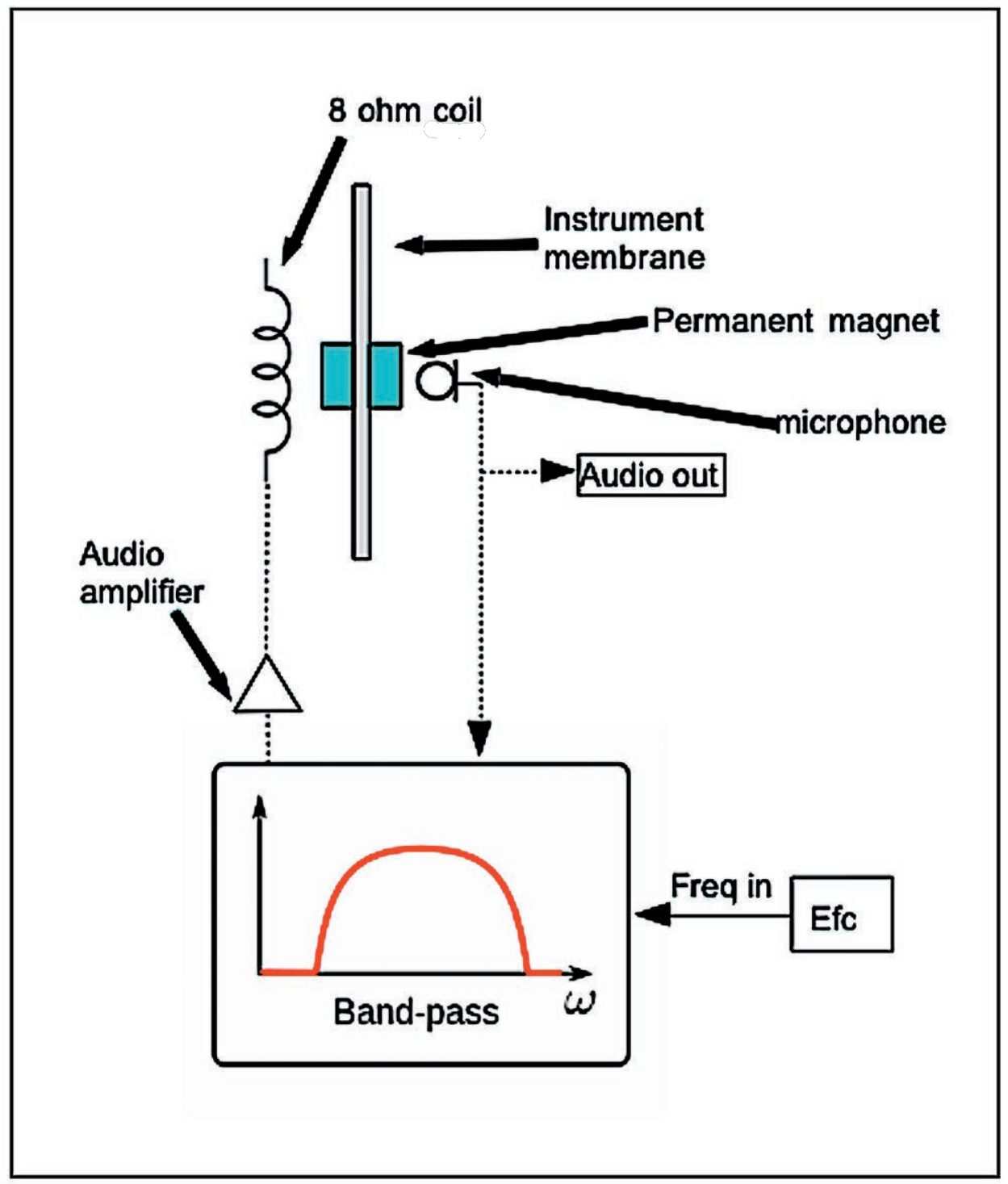

Figura 24. (C) S. Pappalardo, Millis(). An improvisation performance for a garden of remotely controlled mechanical instruments, inedito. Schema di costruzione di uno strumento elettromagnetico.

Un magnete viene fissato al corpo vibrante, ed eccitato grazie al campo elettromagnetico che si viene a creare tre una bobina da $8 \mathrm{ohm}$ amplificata, il magnete sulla superficie risonante, e un microfono auto costruito, filtrato da un filtro passa-banda.

Nel Video 6, Simone Pappalardo illustra la costruzione e il controllo di uno strumento elettromagnetico.

[Video 6 https://zenodo.org/record/4903419] 


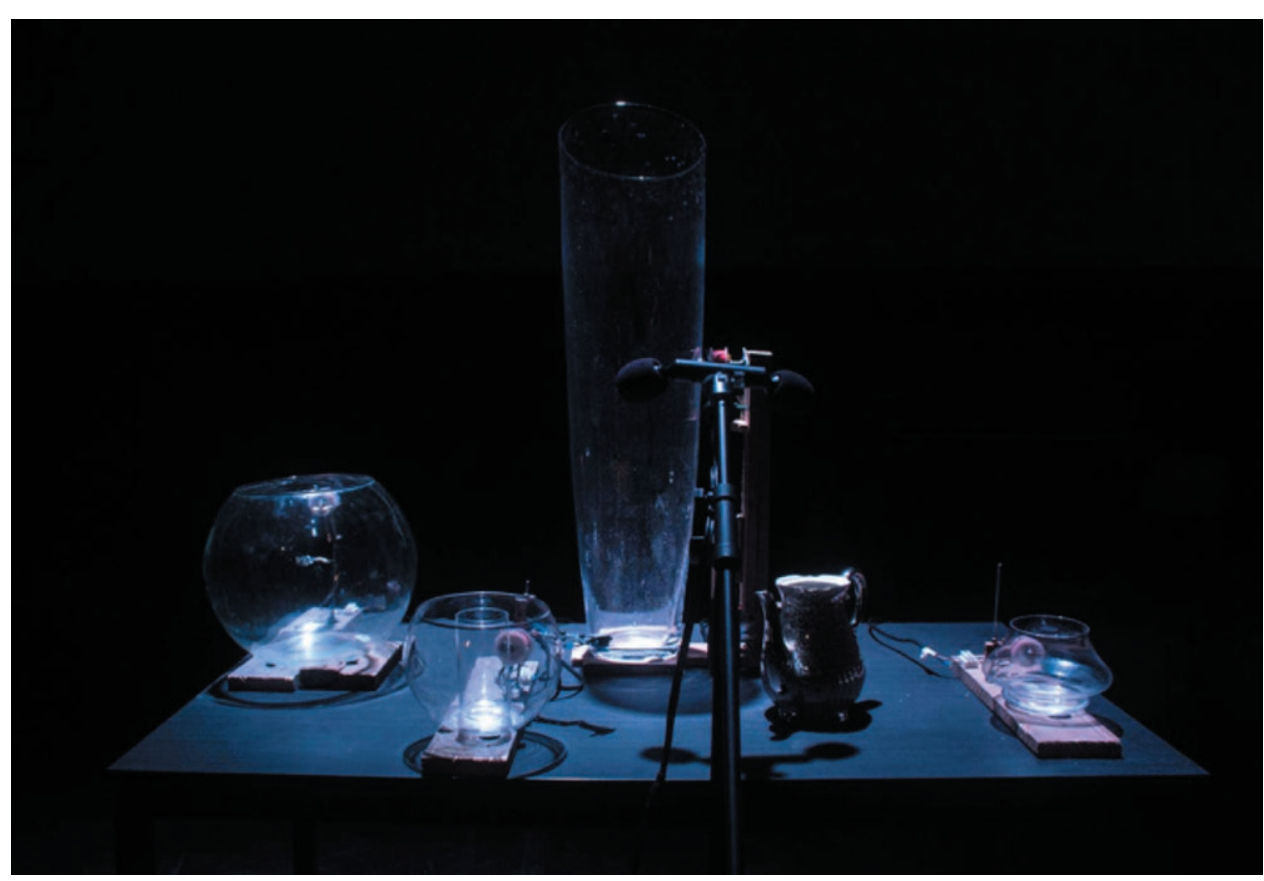

Figura 25. (CS. Pappalardo, alcuni risonatori in vetro di Orchestra Fragile.

Nel caso di Orchestra Fragile (Fig. 25), la componente di filtraggio - che avviene su Max/MSP - è controllata da una serie di descrittori audio che individuano quando la superficie di vetro è sul punto di rottura, riportando la risonanza del corpo ad uno stato che normalizza la sua condizione. La fragilità, come metafora, assume per l'autore diverse connotazioni concettuali:

La rottura è un "momentaneo", uno stato non definitivo; la fragilità è invece un processo in divenire, auto simile, che ricopia sé stesso in forme sempre più larghe, in orchestre organizzate composte di atomi fragili. La malattia è ad esempio un momento di rottura, un punto di arrivo della fragilità, ma le fragilità umane portate alla luce dalla malattia sono solo provvisorie, sposteranno presto i propri confini, lasciando posto a nuovi confini raggiungibili: la guarigione o la morte, la speranza o la rassegnazione, dunque nuove fragilità ${ }^{65}$.

All'opposto del concetto di fragilità troviamo l'idea alla base di Forte (Fig. 26), opera dedicata al paesaggio sonoro e ambientale industriale di Piombino. Nelle parole del compositore:

Il termine "Forte" suggerisce la resistenza del ferro, materiale impiegato per realizzare gli strumenti della performance e produzione industriale principale di Piombino. Forte

$65<$ https://www.goethe.de/ins/it/it/ver/bio/spa.html> 


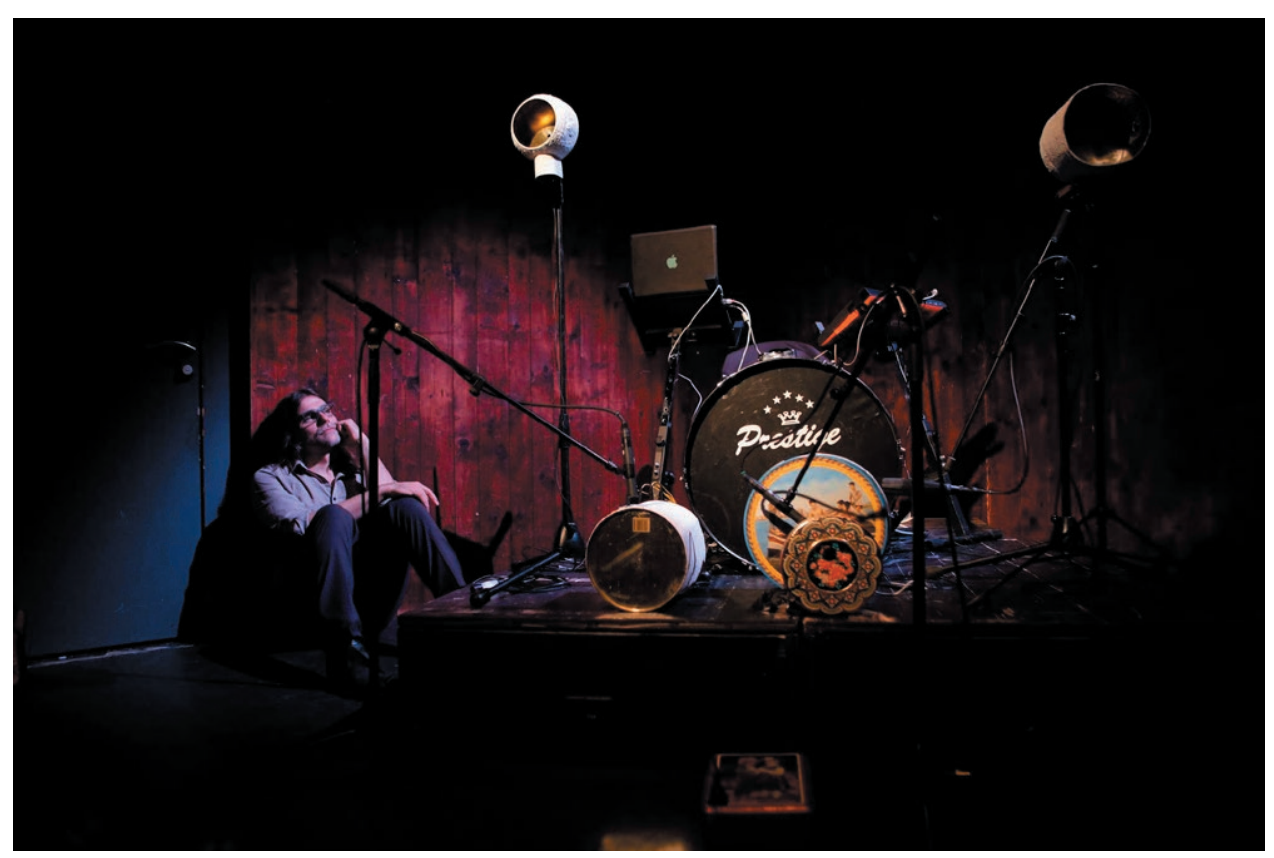

Figura 26. Il compositore Simone Pappalardo, e la versione di Forte presentata al Festival Les Amplitudes. CPablo Fernandez, Festival Les Amplitudes, 2018.

è anche il cognome del noto ricercatore musicale i cui metodi di analisi sono utilizzati durante la performance per produrre in tempo reale le strutture timbriche e musicali. Piombino è in oltre la città in cui vive e ha lavorato per anni mio padre e "Forte", anche nella fragilità della vecchiaia, è il primo aggettivo che mi viene in mente pensando a lui a cui la performance è dedicata ${ }^{66}$.

È interessante come il nome dell'opera si riferisca anche agli studi sulla set theory di Allen Forte ${ }^{67}$, che il compositore utilizza come livello organizzativo dei dati di un complesso algoritmo adattivo. Pappalardo utilizzerà la stessa tecnica in Millis(), indagandola nel dettaglio e piegandola alle sue esigenze musicali.

Gli strumenti elettromagnetici sviluppati in Forte consistono nel sistema di eccitazione già illustrato in Fig. 24, che impiega come corpi di risonanza dei contenitori di metallo di diverse dimensioni.

Un ultimo esempio chiave della produzione di Pappalardo è rappresentato da Millis(), una performance creata in collaborazione con l'artista José Angelino, ispirata ai concetti di teoria del paesaggio di Gilles Clemens ${ }^{68}$, in cui vengono indagati due gruppi strumentali automatizzati, in comunicazione tra di loro. Mentre il parco stru-

$66<$ http://sabinaelettroacustica.it/simone-pappalardo/>

67 A. Forte, The Structures of Atonal Music, Yale University Press, New Haven e Londra 1973.

68 G. Clement, Le Jardin en mouvement. De La vallée au champ via le parc André-Citroën et le jardin planétaire, Sens et Tonka, Parigi 1994. 
mentale di Angelino è analogico, quello di Pappalardo è interamente automatizzato, e racchiude alcuni degli strumenti indagati per l'opera Forte, per il brano The Kempelen Machine di Mauro Lanza, e delle ricerche personali sul recupero di oggetti socialmente storicizzati, come giradischi o violini. Nel caso di Millis() gli oggetti operano sul livello del physical computing, e dell'audio physical computing, indagato e teorizzato da Valle ${ }^{69}$. Gli strumenti che agiscono computazionalmente, controllati da Arduino o dalla microboard wireless ESP $32^{70}$, sono il violino per elisione, l'electromagnetic field synth, e il granular $d j$.

Il violino per elisione consiste in un corpo di violino privato delle sue corde e del ponticello, utilizzato come corpo risonante per sei carillon preparati, e controllati grazie alla micro board Arduino, poi sostituita in tempi recenti con il ESP32 senza fili. Come testimoniato dal compositore e performer, questi sei carillon sono preparati in modo "sottrattivo", secondo tre livelli: originalmente tutti i carillon suonano la melodia del celebre Für Elise di Beethoven, il primo livello di preparazione consiste nel rimuovere le linguette ai carillon in modo da seguire i rapporti numerici della serie di Fibonacci; il secondo stadio di intervento sui carillon consiste nella limatura empirica delle linguette rimanenti, per scordare leggermente le note rimanenti dal primo livello di preparazione; il terzo consiste nell'automazione grazie alla microboard e al controllo con computer. L'electromagnetic field synth consiste in un sintetizzatore basato su Arduino che produce frequenze audio tradotte in campi elettromagnetici, utilizzando un relè come bobina di un altoparlante "silenzioso". I segnali audio vengono innestati in un feedback elettromagnetico, attraverso un microfono auto-costruito utilizzando due relè di ricezione. La tecnica di sintesi sviluppata garantisce una discreta complessità spettrale con un numero limitato di comandi Arduino. Il segnale sonoro e il campo elettromagnetico vengono infine perturbati ancora una volta da ventole del computer, alimentatori in commutazione e altre componenti elettronici di riciclo.

Il granular dj è un giradischi controllato via computer tramite Arduino, dal quale è possibile gestire i parametri di velocità e direzione del piatto rotante, e l'attivazione meccanica di tre testine di lettura, per creare ritmi o suoni granulari asincroni.

Gli strumenti elettromagnetici, oltre agli strumenti già illustrati per Forte, sono i FKinstruments. Il FKinstrument è uno strumento originariamente commissionato e progettato per The Kempelen Machine di Mauro Lanza, consiste in due grandi altoparlanti a tromba a banda larga che si rimodulano a vicenda. Uno dei due altoparlanti riproduce i suoni attraverso una forma di vetro pirex, modellata sulle proporzioni di una radiografia del tratto vocale dell'autore nell'atto di emettere la vocale [i]. Il secondo speaker, attraverso un tubo di rame, modula il flusso d'aria all'interno del risonatore di vetro. La frequenza di attivazione può essere predeterminata o calcolata dinamicamente come risposta al contesto da un algoritmo basato sulla catena di Markov nascosta.

Questi strumenti comunicano attivamente tra di loro attraverso un complesso sistema di generazione e organizzazione di dati, utilizzando algoritmi auto-generativi

69 A. Valle, Sonagraph. A cartoonified spectral model for music composition, in SMC Sound \& Music Computing Conference proceedings 2011, Málaga 2019.

$70<$ http://esp32.net/> 
e tecniche di controllo di sistemi di feedback, formalizzati - e indipendenti - a più livelli dell'opera. La dimensione performativa temporale è costruita intorno alla logica dell'attivazione dei gruppi strumentali automatizzata, continuamente variabile e in movimento. Gli eventi formali che si succedono nei processi di interazione tra le parti di un grande apparato strumentale sono organizzati secondo tecniche algoritmiche ispirate a comportamenti biologici.

L'algoritmo di controllo, il vero e proprio master clock dell'intera performance, è affidato ad un'azione fisica che metaforizza il battito cardiaco biologico: una lastra di metallo automatizzata attraverso un meccanismo di auto-oscillazione regolare (e presente nello strumentario di José Angelino), che invia impulsi generando un segnale di controllo a dente di sega, a sua volta moltiplicato per ottenere sette segnali di controllo sincronico per gli strumenti periferici. Ogni segnale a dente di sega viene quindi tradotto in impulsi, filtrati da una matrice organizzata secondo la logica gerarchica che viene a crearsi utilizzando l'equazione di Verhulst, formula impiegata per descrivere la crescita di una popolazione biologica:

$$
d N / d t=r N(1-N / K)
$$

dove $N$ rappresenta la dimensione della popolazione; $t$ è il tempo; la costante $r$ definisce il tasso di crescita; $K$ è la capacità di portata. Nelle parole del compositore, quindi:

In composition terms it is possible to distinguish $r$ events selected, which will create sudden and non-recursive rhythmic figurations, and $\mathrm{k}$ events selected, whose more or less complex rhythmic patterns will repeat themselves several times with small changes ${ }^{71}$.

In questo modo i valori $r$ (crescita) e $K$ (portata) possono essere gestiti nel momento performativo, o automaticamente attraverso algoritmi ricorsivi che si adattano alla quantità di silenzio rilevato. Un esempio di matrice di controllo ottenuta si riscontra in Fig. 27.

La struttura di comunicazione di dati per controllare gli strumenti, in Millis() si configura con processi di feedback, annidati su più livelli, con processi di transcodifica dei segnali audio in dati numerici, che vengono processati in tre modi distinti ma interconnessi:

- Per riduzione: tecniche algoritmiche storicamente affermate (attrattori, catene di Markov, ma anche operazioni su un pitch set, etc.), vengono redistribuite, attraverso matrici, e utilizzate per gestire localmente alcuni parametri degli strumenti. I processi formali vengono gestiti nel tempo, trasformati e distribuiti ai complessi strumentali per definire microstrutture aperte e possibili di una ridefinizione in tempo reale - automatizzata con processi ricorsivi o controllata dal compositore-performer. Una

71 S. Pappalardo, Millis(). An improvisation performance for a garden of remotely controlled mechanical instruments, inedito, 2020. 


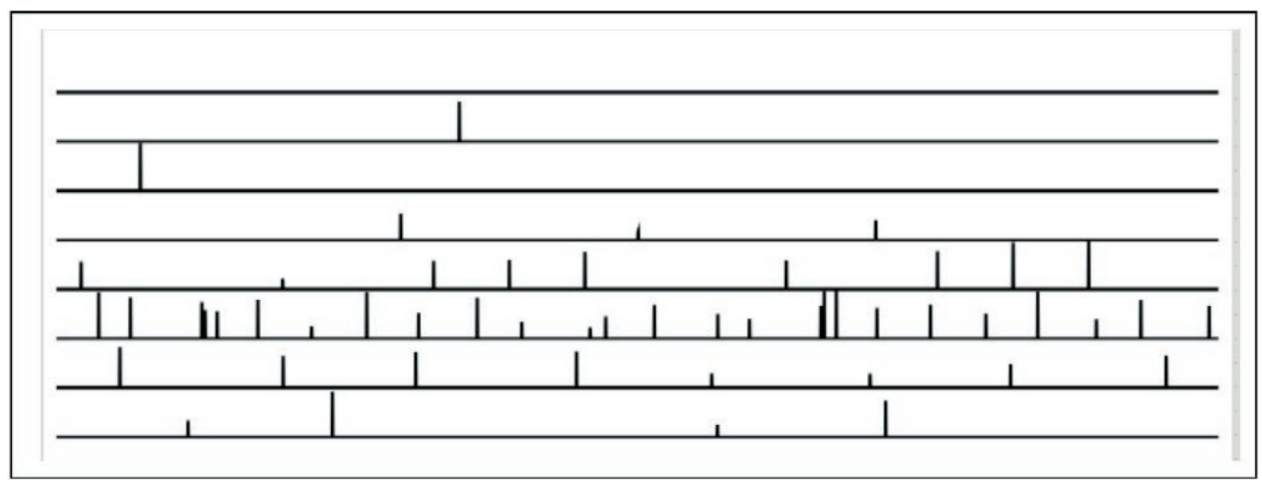

Figura 27. (C) S. Pappalardo, Millis(). An improvisation performance for a garden of remotely controlled mechanical instruments, inedito. Matrice di controllo dei dati. La matrice è creata dai clock generati dall'oscillazione della lastra e filtrati con l'equazione di Verhulst.

caratteristica molto evidente in Millis() è infatti la continua ridefinizione dei processi formali, intesi come materiali musicali.

- Per interconnessione: attraverso l'uso estensivo di tecniche di feedback gestite da matrici annidate. Il feedback influenza sia il timbro degli strumenti musicali automatici che il comportamento degli algoritmi.

- Attraverso l'ostinazione: alcune formalizzazioni, che il compositore chiama "obbligate", sono generate a partire dalle caratteristiche fisiche dei materiali. Una parte degli strumenti in Millis() hanno sistemi di attivazione e logiche di funzionamento non mediati da sistemi digitali. Questi strumenti sviluppano forme temporali elettromeccaniche, basate sulle proprietà elastiche dei materiali o, più in generale, sulle loro caratteristiche fisiche. Questi strumenti si rifanno principalmente allo strumentario di José Angelino, non indagato in questa analisi.

Come nella performance Forte, parte del processo computazionale è svolto da un algoritmo ispirato alla logica della pitch set theory che annovera il teorico americano Allen Forte, appunto, tra i maggiori ricercatori di questo approccio di analisi - che consiste in astrazione numerica a base dodici delle altezze, analizzabili a loro volta applicando logiche dell'analisi insiemistica matematica. Nel caso di Forte e Millis() questa logica di astrazione è applicata al dominio di organizzazione di dati derivati dall'analisi del suono degli strumenti stessi in tempo reale, attraverso un microfono, ed è utilizzata come codifica comune tra gli insiemi strumentali. L'algoritmo sviluppato per l'analisi del suono si basa sul SonaGraph di Andrea Valle illustrato nel paragrafo 4.2, ed è implementato in Max/MSP. Per la conversione e la manipolazione dei set di dati, il compositore ha implementato una propria libreria in javascript per Max/MSP (Forte for live library - ffll). Il convertitore parte dal dato analitico sonoro e lo riporta ad un numero intero a modulo 12, astraendo quindi delle liste che vengono manipolate come insiemi e sottoinsiemi metaforici di una pitch set theory computazionale. Questi insiemi vengono sottoposti a operazioni di inversioni, complementarità, retrogradazione - come i pitch set della musica post- 


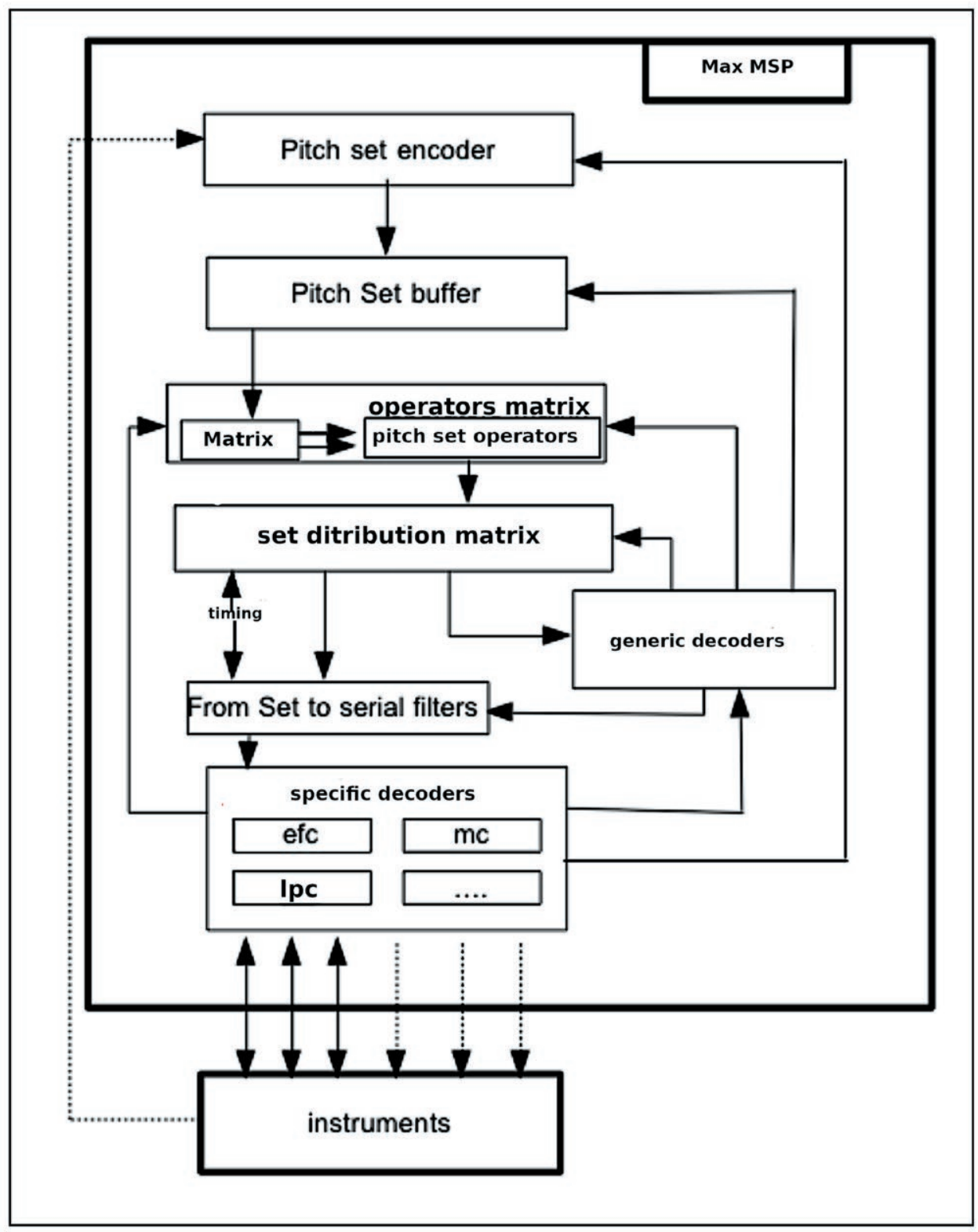

Figura 28. (C) S. Pappalardo, Millis(). An improvisation performance for a garden of remotely controlled mechanical instruments, inedito. Distribuzione dei dati per la performance Millis().

tonale - ma agiscono, convertendosi nelle unità di misura di volta in volta necessarie, nell'organizzazione di parametri strumentali in tempo reale, che influenzano il risultato sonoro complessivo, andando a sua volta a modificare i parametri analitici in tempo reale, quindi ricorsivamente l'organizzazione dei set a base 12 . A loro volta, ogni parametro di questo complesso sistema ricorsivo di comunicazione di dati 
è passibile di modifica da parte del compositore, nonostante la sua stretta relazione con l'intero sistema.

\section{Conclusioni e prospettive}

Nei casi qui indagati l'apparato strumentale automatizzato viene rimodellato in varie forme, creando di volta in volta diversi sistemi con corpi sonori differenti, le cui tecniche di controllo vengono di volta in volta adattate agli strumenti stessi, ma soprattutto alle complesse necessità musicali. Degli apparati strumentali quindi, costruiti con mezzi apparentemente semplici, primitivi, grezzi, e di recupero, che vengono impiegati all'interno di progetti strumentali altamente complessi a livello computazionale e di gestione dei dati algoritmici.

Questo approccio alla semplicità meccanica in relazione alla complessità computazionale, nonostante sia relativamente recente e in via di continua sperimentazione, è stato accolto positivamente anche dai compositori delle generazioni più giovani. Due esempi peculiari sono le produzioni musicali di Alessandro Perini e Johanes Svensson.

Nel caso della produzione più recente di Alessandro Perini, compositore comasco, classe 1983 e residente in Svezia, gli strumenti classicamente intesi vengono automatizzati solo in parte, richiedendo quindi un continuo confronto con la componente performativa umana. Ne sono un esempio i brani composti nel 2020 che formano il trittico per strumento solista e componente automatizzata: Rondó (per chitarra elettrica e piroli controllati via midi) ${ }^{72}$, Epicentro (per pianoforte, dieci motori a vibrazione e due microfoni a contatto) ${ }^{73}$, Intorno alla traccia (per clarinetto con chiavi automatizzate e live electronics $)^{74}$. Il trittico di Perini vede un modello di interazione con lo strumento automatizzato radicalmente differente rispetto a quelli studiati nei casi di Lanza, Valle e Pappalardo. La componente umana in Perini è necessaria per il funzionamento dell'apparato strumentale, ma la sua automazione ne estende le possibilità di intervento tecnico, e quindi timbrico e musicale.

Svensson, infine, integra gli apparati strumentali automatizzati all'interno di un ensemble umano similmente a Lanza e Valle, ma integrando una componente visiva di dispositivi luminosi, riflettendo attivamente sulle qualità visuali come materiale compositivo, e trasformando la performance in una sorta di teatro musicale iper tecnicizzato. Ne sono un esempio Stop Motion (2018, per ensemble, strumenti elettromeccanici e luci), Double Dubbling (firefly song) (2019-2020, per fisarmonica, clarinetto e 16 piezo buzzers).

\footnotetext{
$72<$ https://alessandroperini.com/2021/01/25/rondo/>

$73<$ https://alessandroperini.com/2021/02/03/epicentro/>

74 <https://alessandroperini.com/2021/02/08/intornoallatraccia/>
} 


\section{Bibliografia}

Agon, C., Bresson J. and Assayag G. (a cura di), The OM Composer's book vol. 1-2-3, Editions Delatour France/IRCAM, Parigi 2006.

Albert, G. 'Sound sculptures' e 'sound installations', «AAA - TAC», 7, Fondazione Giorgio Cini Venezia, Istituto per la musica, Fabrizio Serra editore, Roma-Pisa 2010.

Assayag, G. and Rueda, C. Computer-Assisted Composition at IRCAM: From PatchWork to OpenMusic, "Computer Music Journal», vol. 23, no. 3, 1999, 59-72.

Banzi, M. Getting started with Arduino. O’Reilly, 2009.

Bastani, A. Fully Automated Luxury Communism, Verso, Londra 2019.

Battier, M. (a cura di), Modalys. An introduction, IRCAM, Parigi 1997 (ed. orig. 1991).

Bauer, A. Automata in extremis: Mauro Lanza's sublime sound machines, "Nuove musiche», no. 5, 2021.

Boulanger, R. (a cura di), The Csound Book, The MIT Press, Cambridge 2000.

Collins, N., Wilson, S. and Cottle, D. (a cura di), The SuperCollider Book. The MIT Press, Cambridge 2011.

Collins, N., Schedel, M. and Wilson, S. (a cura di), Electronic Music, Cambridge Introductions to Music, Cambridge University Press, Cambridge 2013.

Clement, G. Le Jardin en mouvement. De La vallée au champ via le parc André-Citroën et le jardin planétaire, Sens et Tonka, Parigi 1994.

Fantechi, D., Systema Naturae, by Andrea Valle and Mauro Lanza, experimentation as starting point of a piece of "acoustic computer music", "Proceedings of the Electroacoustic Music Studies Network Conference», Firenze 2018.

Fineberg, J. Guide to The Basic Concepts and Techniques Of Spectral Music, «Contemporary Music Review», 19:2, 2000, pp. 81-113.

Foglia, C. Ristrutturazione spettrale di armonie funzionali: l'evoluzione della forma teleologica in Kaija Saariaho, tesi di laurea magistrale, Università di Pavia - Dipartimento di Musicologia e Beni Culturali, Cremona 2020.

Forte, A. The Structures of Atonal Music, Yale University Press, New Haven e Londra 1973.

Galpin, F. A Textbook of European Musical Instruments. William, London 1937.

Ghazala, R. Circuit-Bending. Build Your Own Alien Instruments. Wiley, Indianapolis 2005.

V. Hornbostel, E. M. and Sachs, C. Classification of musical instruments, «The Galpin Society Journal», 14, 1961, pp. 3-29.

Hopkin, B. Musical instrument design. Practical information for instrument making, Sea Sharp Press, Tucson 1996.

Hufschmitt, A. La synthese par modeles physiques, tesi di dottorato, Université de Paris Sorbonne (Paris IV) U.F.R. de Musique et Musicologie, Parigi 2000.

Kartomi, M. The classification of musical instruments: Changing trends in research from the late nineteenth century, with special reference to the 1990s, "Ethnomusicology», vol. 45, no. 2, 2001, pp. 283-314.

Lanza, M. note di sala del concert-atelier monografico dedicato a Mauro Lanza, IRCAM, Espace de projection, Parigi, 13/02/2004.

Lanza, M., Verlingieri, G., Biagioni, N. La libreria OpenMusic om4Csound, in A. Valle and S. Bassanese (a cura di), "Prossime distanze. Atti del XVIII CIM», Edizioni AIMI, Venezia 2011. 
Magnusson, T. Sonic Writing. Technologies of material, symbolic and signal inscriptions, Bloomsbury Academic, New York 2019.

Mallozzi, C. e Tortora, D. (a cura di), La bottega del suono. Mario Bertoncini. Maestri e allievi, Editoriale Scientifica, Napoli 2017.

Manca, G., Manfrin, L. (a cura di), Fare Strumento. Composizione, invenzione del suono e nuova liuteria, Edizioni ETS, Pisa 2018.

Mathews, M. (a cura di), The Technology of Computer Music, MIT Press, Cambridge 1969.

Manovich, L. The Language of New Media, MIT Press, Cambridge Mass., 2001.

O’Sullivan, D. and Igoe, T. Physical Computing, Course Technology, Boston 2004.

Panariello, C. Study in three phases. An Adaptive Sound Installation, in «Leonardo Music Journal», 30, 2020, pp. 44-49.

Pappalardo, S. Millis(). An improvisation performance for a garden of remotely controlled mechanical instruments, inedito, 2020.

Patteson, T. Instruments for new music. Sound, technology, and modernism, University of California Press, Oakland 2016.

Puckette, M. The Theory and Technique of Electronic Music, World Scientific Publishng Co. Inc., River Edge 2007.

Pustijanac, I. Oralità 'digitalizzata' nelle edizioni del repertorio contemporaneo, in "Convegno: Filologia musicale e tecnologie digitali a colloquio con la prassi esecutiva"», Fondazione Guido d'Arezzo, Arezzo/online 2020.

Roads, C. (a cura di), The Computer Music Tutorial, MIT Press, Cambridge 1996.

Rocchesso, D. e Fontana, F. (a cura di), The Sounding Object, Edizioni di Mondo Estremo, 2003.

Roullier, P. (a cura di), Mauro Lanza et Andrea Valle: Systema naturae. Ensemble 2e2m, Champigny-sur-Marne 2016.

Sachs, C. The History of Musical Instruments, Norton, New York 1940

Valle, A. and Lanza, M. Systema naturae: shared practices between physical computing and algorithmic composition, in J. P. Tapio Lokki and V. Välimäki (a cura di), «Proceedings of the 14th Sound and Music Computing Conference», Aalto University, Espoo 2017, pp. 391-398.

Valle, A. e Lombardo, V. Audio e Multimedia, Maggioli, Santarcangelo di Romagna 2014 (ed. orig. Apogeo, Milano 2002).

Valle, A. and Pappalardo, S. Electric grammars. Algorithmic design and construction of experimental music circuits, «Proceedings of the 14th Sound and Music Computing Conference», Espoo 2017, pp. 351-358.

Valle, A. and Pappalardo, S. The Gate Modulator. An experiment in digitally-controlled analog synthesis, "Atti del XX CIM - Colloquio di Informatica Musicale», Roma 2014.

Valle, A. and Sanfilippo, D. Feedback Systems: An Analytical Framework, "Computer Music Journal», vol. 37, no. 2, 2013, pp. 12-27.

Valle, A. Audio physical computing, in SMC Sound \& Music Computing Conference proceedings 2011, Padova 2011.

, La notazione musicale contemporanea. Aspetti semiotici ed estetici, De Sono-EDT, Torino 2002 .

, Integrated Algorithmic Composition. Fluid Systems for including notation in music composition cycle, «NIME: Proceedings», 2008, pp. 253-256. 
, Notazioni elettromeccaniche, o forse no, «Musica/Tecnologia», no. 13, Firenze University press, Firenze 2019.

, Making Acoustic Computer Music: The Rumentarium project, in Organised Sound, 18(03), Cambridge 2013, pp 242-254.

, Introduzione a SuperCollider Apogeo Education - Maggioli Editore, Milano 2015.

, Residual orchestras: Notes on low profile, automated sound instruments, in Proc. of the Cumulus Conf. '15, McGraw-Hill, Milano 2015, pp. 717-729.

, SampComp: sample-based techniques for algorithmic composition, in Proceedings of the 22nd CIM, Udine 2018, pp. 128-135.

, Sonagraph. A cartoonified spectral model for music composition, in SMC Sound \& Music Computing Conference proceedings 2011, Málaga 2019.

Verrando, G. Gli strumenti come apparati, 2016. <https://www.giovanniverrando.net/lutheriecomposition/gli-strumenti-come-apparati/>

Verrando, G. et al. (a cura di), La nuova Liuteria: orchestrazione, grammatica, estetca, Suvini Zerboni, Milano 2012.

Vinet, H. Recent Research and Development at IRCAM, "Computer Music Journal», vol. 23, no. 3, 1999, pp. 9-17.

Žižek, S. (a cura di), Mapping Ideology, Verso, Londra 1994.

\section{Sitografia (ultima consultazione 12.03.2021)}

https://andreavalle.bandcamp.com/

https://soundcloud.com/maurolanza

https://vimeo.com/244839789

http://arnofabre.free.fr/en/Dropper01/Dropper01.html

http://musiquealgorithmique.fr/entretien-6-andrea-valle/

https://www.soundesign.info/2009/05/20/intervista-con-andrea-valle/

https://matiere-memoire.bandcamp.com/album/mmxx-14-fully-automated-luxury-communism https://supercollider.github.io/

https://openmusic-project.github.io/

https://cycling74.com/products/max

http://www.thenewnoise.it/i-regnum-di-andrea-valle-e-mauro-lanza/

https://www.musicaelettronica.it/come-costruire-un-serraturofono-a-borracce-programmabilee-interattivo-guida-teorico-pratica-1/

https:/www.musicaelettronica.it/come-costruire-un-serraturofono-a-borracce-programmabilee-interattivo-guida-teorico-pratica-2/

https://www.musicaelettronica.it/come-costruire-un-serraturofono-a-borracce-programmabilee-interattivo-guida-teorico-pratica-3/

https:/www.musicaelettronica.it/campcomp-lineamenti-di-musica-audiopara-

https://www.musicaelettronica.it/campcomp-lineamenti-di-musica-audiopara-2/

https://www.musicaelettronica.it/spettri-fourier-e-il-geco-1/ 
https://www.musicaelettronica.it/spettri-fourier-e-il-geco-2/

https://www.musicaelettronica.it/osservazioni-di-genetica-del-ritmo/

http://www.musicaelettronica.it/osservazioni-di-genetica-del-ritmo-2/

https://forum.ircam.fr/projects/detail/modalys/

http://support.ircam.fr/docs/Modalys/current/co/publication-web.html

https://www.ircam.fr/recherche/equipes-recherche/systemes-et-signaux-sonores-audioacoustique-instruments-s3am/

https://github.com/vanderaalle

http://support.ircam.fr/docs/AudioSculpt/3.0/co/AudioSculptguideWeb.html

https://medias.ircam.fr/embed/media/xd098bc_the-making-of-ludus-de-morte-regis-vf

https://www.ilsaxofonoitaliano.it/artisti/pappalardo-simone/

http://sabinaelettroacustica.it/simone-pappalardo/

https://www.goethe.de/ins/it/it/ver/bio/spa.html

https:/www.fluid-radio.co.uk/2013/05/postcards-from-italy-rome-simone-pappalardo/

https://www.artribune.com/mostre-evento-arte/jose-angelino-e-simone-pappalardo-millis/

https://www.facebook.com/793985660723793/videos/396656014901634

https://medias.ircam.fr/x58ad83

https://medias.ircam.fr/x4179ef

https://alessandroperini.com 